\title{
SUPPORT VARIETIES, AR-COMPONENTS, AND GOOD FILTRATIONS
}

\author{
ROLF FARNSTEINER and GERHARD RÖHRLE
}

\begin{abstract}
Let $G$ be a reductive group, defined over the Galois field $\mathbb{F}_{p}$ with $p$ being good for $G$. Using support varieties and covering techniques based on $G_{r} T$-modules, we determine the position of simple modules and baby Verma modules within the stable Auslander-Reiten quiver $\Gamma_{s}\left(G_{r}\right)$ of the $r$-th Frobenius kernel of $G$. In particular, we show that the almost split sequences terminating in these modules usually have an indecomposable middle term.

Concerning support varieties, we introduce a reduction technique leading to isomorphisms

$$
\mathcal{V}_{G_{r}}\left(Z_{r}(\lambda)\right) \cong \mathcal{V}_{G_{r-d}}\left(Z_{r-d}(\mu)\right)
$$
\end{abstract}

for baby Verma modules of certain highest weights $\lambda, \mu \in X(T)$, which are related by the notion of depth.

\section{INTRODUCTION}

In the representation theory of finite-dimensional self-injective algebras, the stable AuslanderReiten quiver has proven to be an important homological invariant, which has been studied for group algebras of finite groups, reduced enveloping algebras of restricted Lie algebras and distribution algebras of infinitesimal group schemes. In the classical context of Frobenius kernels of reductive groups, the relevant algebras are usually of wild representation type, rendering a classification of their indecomposable modules a hopeless task. This fact notwithstanding, one does have a fairly good understanding of the connected components of the corresponding stable Auslander-Reiten quiver. It is therefore of interest to relate this information to certain classes of indecomposable modules, such as simple modules, Weyl modules or Verma modules, and to identify the position of the latter within the AR-quiver. The main problem in this undertaking is the lack of a suitable presentation of the underlying algebras, as required by the techniques of abstract representation theory. On the other hand, the theory of rank varieties and support varieties has seen considerable progress over the last years, so that one can hope to exploit these tools in the aforementioned context.

In continuation of work begun in [22, we study in this article those Auslander-Reiten components of the algebras $\operatorname{Dist}\left(G_{r}\right)$ that contain simple modules or baby Verma modules. The underlying algebra Dist $\left(G_{r}\right)$ consists of the distributions of the $r$-th Frobenius kernel of the smooth reductive group scheme $G$, defined over an algebraically closed field of positive characteristic $p$. The case $r=1$, which pertains to restricted enveloping algebras of reductive Lie algebras, was settled in 22 by means of a detailed analysis of nilpotent orbits in rank varieties, leading to the consideration of groups of types $\mathrm{SL}(2)_{1} \times \mathrm{SL}(2)_{1}, \mathrm{SL}(3)_{1}$ and $\mathrm{SO}(5)_{1}$. For $r>1$, rank varieties are less tractable and the structure of the cohomology rings defining support varieties is more complicated. We address these problems by passage to $G_{r} T$-modules, as described below. With regard to Auslander-Reiten theory, our main results can roughly be summarized as follows:

Theorem. Let $G$ be a smooth reductive group scheme, defined over $\mathbb{F}_{p}$. Given a character $\lambda \in X(T)$ and $r \geq 1$, the following statements hold:

Date: June 22, 2018.

2000 Mathematics Subject Classification. Primary 16G70, Secondary 17B50. 
(1) The simple $G_{r}$-module $L_{r}(\lambda)$ is either projective or it belongs to an AR-component of tree class $\tilde{A}_{12}$ or $A_{\infty}$. In the latter case, the middle term of the almost split sequence terminating in $L_{r}(\lambda)$ is indecomposable.

(2) The baby Verma module $Z_{r}(\lambda)$ is either projective or it belongs to an AR-component of tree class $A_{\infty}$, with the middle term of the almost split sequence terminating in $Z_{r}(\lambda)$ being indecomposable.

The approach chosen in this article differs from that of [22] by the systematic use of Jantzen's category $\bmod G_{r} T$ of $G_{r} T$-modules, which affords almost split sequences. Exploiting the natural ordering on the weights $X(T)$, we first study AR-components for $G_{r} T$-modules, and then use covering properties of the forgetful functor $\mathfrak{F}: \bmod G_{r} T \longrightarrow \bmod G_{r}$ to obtain information on the corresponding $G_{r}$-modules. The second major advantage of working in $\bmod G_{r} T$ is its tractability with respect to coverings $\tilde{G} \longrightarrow G$, ultimately allowing us to bring Steinberg's tensor product theorem to bear. Aside from these technical aspects, the highest weight category $\bmod G_{r} T$ possesses the much studied subcategory $\mathcal{F}(\Delta)$ of $\widehat{Z}_{r}$-filtered modules. According to Ringel's seminal work [46], the corresponding categories of $\Delta$-good modules over finite-dimensional quasi-hereditary algebras afford relative almost split sequences. It turns out that in our context these are merely the ARsequences of the ambient Frobenius category $\bmod G_{r} T$.

Our paper is organized as follows. After a preliminary section, we determine in Section 2 the location of the simple $G_{r}$-modules. For finite groups and restricted enveloping algebras the corresponding problems were studied in [36, 37, 38, 39] and [16], respectively. In fact, since the algebra $\operatorname{Dist}\left(G_{r}\right)$ is symmetric, Kawata's results [37, that were inspired by the modular representation theory of finite groups, can be brought to bear. With the exception of simple modules belonging to blocks of tame representation type, the AR-components containing simple modules are of type $\mathbb{Z}\left[A_{\infty}\right]$, with the simple vertex having only one predecessor (see part (1) of the above Theorem).

In Section 3 we recall basic results from [19] concerning the AR-Theory of the Frobenius category $\bmod G_{r} T$. As a first application, we exploit coverings $\tilde{G} \longrightarrow G$ to show that each AR-component of $\bmod G_{r}$ contains at most one simple module. Using fundamental properties of the highest weight category $\bmod G_{r} T$, we verify in Section 4 the second part of the above Theorem (cf. [52, Thm. B] for the corresponding result concerning trivial source modules over finite groups).

Sections 5 and 6 are concerned with filtrations and varieties of Verma modules. Motivated by Jantzen's work on blocks of $\operatorname{Dist}\left(G_{r}\right)$ 32, we subdivide the defining highest weights of Verma modules according to their "depth". It turns out that questions concerning varieties and ARcomponents of Verma modules can often be reduced to the consideration of highest weights of depth 1. Our method is based on an isomorphism

$$
\mathcal{V}_{G_{r}}\left(M^{[d]} \otimes_{k} \mathrm{St}_{d}\right) \cong \mathcal{V}_{G_{r-d}}(M),
$$

relating the variety of a $G_{r-d}$-module $M$ to that of the tensor product $M^{[d]} \otimes_{k} \mathrm{St}_{d}$ of its $d$-th Frobenius twist $M^{[d]} \in \bmod G_{r}$ with the $d$-th Steinberg module $\mathrm{St}_{d}$. When applied to baby Verma modules, this yields explicit information on the varieties $\mathcal{V}_{G_{r}}\left(Z_{r}(\lambda)\right)$.

Our final Section 7 illustrates the utility of our results and techniques by providing applications concerning Verma modules of complexity at most 2. For instance, the supports of Verma modules of complexity 2 are shown to be equidimensional.

\section{PRELIMINARIES}

Throughout, we will be working over an algebraically closed field $k$ of characteristic $p \geq 3$. Unless mentioned otherwise, all algebras and modules are assumed to be finite-dimensional. 
Let $G$ be a connected, reductive, smooth group scheme over $k$ with maximal torus $T$ and character group $X(T)$. We fix a Borel subgroup $B=T U$ with unipotent radical $U$ and denote the Lie algebra of $U$ by $\mathfrak{u}=\operatorname{Lie}(U)$. Moreover, we write $\Psi=\Psi(T), \Psi^{+}$and $\Sigma$ for the set of roots of $G$ relative to $T$, the sets of positive and simple roots of $\Psi$ relative to $B$, respectively.

For $\lambda \in X(T)$ and $r \in \mathbb{N}$ we put

$$
\Psi_{\lambda}^{r}:=\left\{\alpha \in \Psi \mid\left\langle\lambda+\rho, \alpha^{\vee}\right\rangle \in p^{r} \mathbb{Z}\right\},
$$

where $\rho:=\frac{1}{2} \sum_{\alpha \in \Psi^{+}} \alpha$ denotes the half-sum of the positive roots. We say that $\lambda$ is $p^{r}$-regular (relative to $\Psi$ ) provided $\Psi_{\lambda}^{r}=\varnothing$. For $r=1$ we simply write $\Psi_{\lambda}=\Psi_{\lambda}^{1}$. The set of $p^{r}$-restricted weights $X_{r}(T)$ of $T$ is defined by

$$
X_{r}(T):=\left\{\lambda \in X(T) \mid 0 \leq\left\langle\lambda, \alpha^{\vee}\right\rangle<p^{r} ; \alpha \in \Sigma\right\} .
$$

For $G=\mathrm{SL}(2)$ we have $X(T) \cong \mathbb{Z}$ and thus identify weights with integers in this case.

Given $r \in \mathbb{N}$, we let $F^{r}: G \longrightarrow G^{(r)}$ be the $r$-th Frobenius homomorphism of $G$, whose comorphism is

$$
k[G]^{(r)} \longrightarrow k[G] ; x \mapsto x^{p^{r}} .
$$

As usual, $k[G]^{(r)}$ denotes the $k$-algebra, whose underlying $\mathbb{Z}$-algebra structure is that given by $k[G]$, with $k$ acting via $\alpha \cdot x:=\alpha^{p^{-r}} x$ (cf. [35, (I.9)]). The infinitesimal algebraic $k$-group $G_{r}:=\operatorname{ker} F^{r}$ is called the $r$-th Frobenius kernel of $G$.

For a $G_{r}$-module $M$ we define its cohomological support variety $\mathcal{V}_{G_{r}}(M)$ as the variety of the kernel of the canonical homomorphism

$$
\mathrm{H}^{\mathrm{ev}}\left(G_{r}, k\right) \longrightarrow \operatorname{Ext}_{G_{r}}^{\mathrm{ev}}(M, M),
$$

see [51]. We consider the $r$-th Frobenius kernel $\mathbb{G}_{a(r)}:=\operatorname{Spec}_{k}\left(k[T] /\left(T^{p^{r}}\right)\right)$ of the additive group $\mathbb{G}_{a} \cong \operatorname{Spec}_{k}(k[T])$ and recall that

$$
\operatorname{Dist}\left(\mathbb{G}_{a(r)}\right) \cong k\left[X_{0}, \ldots, X_{r-1}\right] /\left(X_{0}^{p}, \ldots, X_{r-1}^{p}\right),
$$

with $X_{i}$ corresponding to the functional $x_{i} \in \operatorname{Dist}\left(\mathbb{G}_{a(r)}\right)=k\left[\mathbb{G}_{a(r)}\right]^{*}$ given by

$$
x_{i}\left(T^{j}+\left(T^{p^{r}}\right)\right)=\delta_{p^{i} j} .
$$

In particular, the algebra $\operatorname{Dist}\left(\mathbb{G}_{a(r)}\right)=k\left[x_{1}, \ldots, x_{r}\right]$ is generated by the $x_{i}$. Owing to [51, (6.8)], the support variety of $M$ is homeomorphic to the rank variety

$$
V_{r}(G)_{M}:=\left\{\varphi: \mathbb{G}_{a(r)} \longrightarrow G|M|_{A_{r}} \text { is not projective }\right\} .
$$

Here $\left.M\right|_{A_{r}}$ denotes the pull-back of the module structure of $M$ along the restriction to $A_{r}:=k\left[x_{r-1}\right]$ of the homomorphism $\operatorname{Dist}\left(\mathbb{G}_{a(r)}\right) \longrightarrow \operatorname{Dist}\left(G_{r}\right)$ corresponding to $\varphi$. In [51] the authors consider the support scheme $V_{r}(G)_{M}$, whose $k$-rational points are our variety $V_{r}(G)_{M}$.

By general theory, the category of $\bmod G_{r}$ of $G_{r}$-modules is equivalent to the module category $\bmod \operatorname{Dist}\left(G_{r}\right)$ of the Hopf algebra $\operatorname{Dist}\left(G_{r}\right)$ of distributions of $G_{r}$, see [35, I, §8]. According to [12, (II, $\S 7,4.2)$ ], the Hopf algebra $\operatorname{Dist}\left(G_{1}\right)$ is isomorphic to the restricted enveloping algebra $U_{0}(\mathfrak{g})$ of the restricted Lie algebra $\mathfrak{g}=\operatorname{Lie}(G)$ of $G$. This isomorphism induces an isomorphism between $\mathcal{V}_{G_{1}}(M)$ and the corresponding variety $\mathcal{V}_{\mathfrak{g}}(M)$ of the $\mathfrak{g}$-module associated to $M$. We shall henceforth identify these two varieties without further notice.

According to [51, (5.4)], a closed embedding $H \hookrightarrow H^{\prime}$ of infinitesimal group schemes gives rise to an embedding $\mathcal{V}_{H}(k) \hookrightarrow \mathcal{V}_{H^{\prime}}(k)$ that maps $\mathcal{V}_{H}(k)$ homeomorphically onto its image. We use this identification and write

$$
\mathcal{V}_{H}(M)=\mathcal{V}_{H^{\prime}}(M) \cap \mathcal{V}_{H}(k)
$$

for an $H^{\prime}$-module $M$. For future reference we record the following well-known fact concerning varieties of relatively projective modules, see for instance [41, (2.3.1)]. 
Proposition 1.1. Let $H \subseteq H^{\prime}$ be infinitesimal group schemes.

(1) If $M$ is a $\left(\operatorname{Dist}\left(H^{\prime}\right): \operatorname{Dist}(H)\right)$-projective $H$-module, then $\mathcal{V}_{H^{\prime}}(M)=\mathcal{V}_{H}(M)$.

(2) If $N$ is an $H$-module, then $\mathcal{V}_{H^{\prime}}\left(\operatorname{Dist}\left(H^{\prime}\right) \otimes_{\operatorname{Dist}(H)} N\right) \subseteq \mathcal{V}_{H}(N)$.

(3) $A \operatorname{Dist}(H)$-module $M$ is projective if and only if $\mathcal{V}_{H}(M)=\{0\}$.

We let

$$
X(T)_{+}:=\left\{\lambda \in X(T) \mid\left\langle\lambda, \alpha^{\vee}\right\rangle \geq 0 \quad \forall \alpha \in \Sigma\right\}
$$

be the set of dominant weights and recall that for every $\lambda \in X(T)_{+}$there exists a simple $G$ module $L(\lambda)$ of highest weight $\lambda$. Moreover, the $L(\lambda)$ form a complete set of representatives for the isoclasses of the simple $G$-modules. Similarly, the simple $G_{r}$-modules are of the form $L_{r}(\lambda)$, with $\lambda$ belonging to a set of representatives for $X(T) / p^{r} X(T)$ (cf. [35, (II.3.10)]). Finally, the simple $G_{r} T$-modules are given by $\widehat{L}_{r}(\lambda)$, with $\lambda \in X(T)$. Note that $\left.\widehat{L}_{r}(\lambda)\right|_{G_{r}} \cong L_{r}(\lambda)$ (see [35, (II.9.6)]).

Given $\lambda \in X(T)$ and $r \in \mathbb{N}$, we let

$$
Z_{r}(\lambda):=\operatorname{Dist}\left(G_{r}\right) \otimes_{\operatorname{Dist}\left(B_{r}\right)} k_{\lambda}
$$

be the (baby) Verma module of $G_{r}$ with highest weight $\lambda[1]$ For a Levi subgroup $L \supseteq T$ of $G$ we define the (baby) Verma module of $L_{r}$ with highest weight $\lambda$ by

$$
Z_{r}^{L}(\lambda):=\operatorname{Dist}\left(L_{r}\right) \otimes_{\operatorname{Dist}\left((B \cap L)_{r}\right)} k_{\lambda} .
$$

We record the following basic result concerning varieties (cf. [41, (2.3.1),(4.2.1)]):

Proposition 1.2. Let $\lambda \in X(T)$. Then the following statements hold:

(1) $\mathcal{V}_{G_{r}}\left(Z_{r}(\lambda)\right) \subseteq \mathcal{V}_{U_{r}}(k)$.

(2) $\mathcal{V}_{L_{r}}\left(Z_{r}^{L}(\lambda)\right) \subseteq \mathcal{V}_{G_{r}}\left(Z_{r}(\lambda)\right)$.

We denote by $W$ and $W_{p}$ the Weyl group and the affine Weyl group associated to the reductive group scheme $G$, respectively. The "dot" action of $w \in W_{p}$ on $X(T)$ is defined as follows:

$$
w \cdot \lambda:=w(\lambda+\rho)-\rho .
$$

Let $H$ be an infinitesimal group scheme, $M$ be an $H$-module. By definition, the complexity $\mathrm{cx}_{H}(M)$ of $M$ is the polynomial rate of growth of a minimal projective resolution $\left(P_{i}\right)_{i \geq 0}$ of $M$, i.e.,

$$
\operatorname{cx}_{H}(M):=\min \left\{n \in \mathbb{N}_{0} \cup\{\infty\} \mid \exists c>0 \operatorname{dim}_{k} P_{i} \leq c i^{n-1} \text { for all } i \geq 1\right\} .
$$

The reader is referred to [4, (5.3)] for basic properties of this notion.

Let $h=h(G)$ denote the Coxeter number of $G$, that is, the maximum of the Coxeter numbers of the simple components of the derived group $(G, G)$.

Recall that a prime $p$ is said to be good for $G$, provided it does not divide any of the coefficients occurring when expressing any root of $G$ as a linear combination of simple roots. Owing to 33 , $(2.7)], \Psi_{\lambda}^{r}$ is a subsystem of $\Psi$ whenever $p$ is good for $G$.

\footnotetext{
${ }^{1}$ Our notation differs from that in Jantzen's book 35, whose notational conventions we follow fairly closely otherwise. In [35] our group $B$ is denoted $B^{+}$.
} 


\section{Auslander-Reiten Components of Simple $G_{r}$-Modules}

Given a self-injective algebra $\Lambda$, we denote by $\Gamma_{s}(\Lambda)$ the stable Auslander-Reiten quiver of $\Lambda$. By definition, the directed graph $\Gamma_{s}(\Lambda)$ has as vertices the non-projective indecomposable $\Lambda$-modules and its arrows are defined via the so-called irreducible morphisms. We refer the interested reader to [2, Chap. VII] for further details. The AR-quiver is fitted with an automorphism $\tau_{\Lambda}$, the so-called Auslander-Reiten translation. Since $\Lambda$ is self-injective, $\tau_{\Lambda}$ coincides with the composite $\Omega_{\Lambda}^{2} \circ \nu_{\Lambda}$ of the square of the Heller translate $\Omega_{\Lambda}$ and the Nakayama functor $\nu_{\Lambda}$, cf. [2, (IV.3.7)].

The connected components of $\Gamma_{s}(\Lambda)$ are connected stable translation quivers. By work of Riedtmann [44, Struktursatz], the structure of such a quiver $\Theta$ is determined by a directed tree $T_{\Theta}$ and an admissible group $\Pi \subseteq \operatorname{Aut}_{k}\left(\mathbb{Z}\left[T_{\Theta}\right]\right)$, giving rise to an isomorphism

$$
\Theta \cong \mathbb{Z}\left[T_{\Theta}\right] / \Pi
$$

of stable translation quivers. The undirected tree $\bar{T}_{\Theta}$ of $T_{\Theta}$ is uniquely determined by $\Theta$ and is called the tree class of $\Theta$. We refer the reader to [3, (4.15.6)] for further details. For group algebras of finite groups, the possible tree classes and admissible groups were first determined by Webb [55], with refinements provided in [47, 42, 5, 6, 14].

Throughout, $G$ is assumed to be a connected smooth reductive algebraic group scheme. By work of Larson and Sweedler [40, every finite-dimensional Hopf algebra is a Frobenius algebra. Consequently, the algebra $\operatorname{Dist}\left(G_{r}\right)$ is self-injective, so that the classes of projective and injective $G_{r}$-modules coincide.

Following Ringel [45], an indecomposable $G_{r}$-module $M$ is called quasi-simple provided its isomorphism class $[M]$ lies at the end of a component of tree class $A_{\infty}$ of the stable AR-quiver $\Gamma_{s}\left(G_{r}\right)$ associated to $\operatorname{Dist}\left(G_{r}\right)$. Given $\lambda \in X(T)$, let $P_{r}(\lambda)$ denote the projective cover of the non-projective simple $G_{r}$-module $L_{r}(\lambda)$ and let $\mathrm{Ht}_{r}(\lambda)=\operatorname{Rad}\left(P_{r}(\lambda)\right) / \operatorname{Soc}\left(P_{r}(\lambda)\right)$ be its heart. We write $\operatorname{Top}(M):=M / \operatorname{Rad}(M)$ for the top of a $G_{r}$-module $M$.

For future reference we record the following basic property of the algebras of distributions (see [48, 30] for related work):

Lemma 2.1. The Hopf algebra $\operatorname{Dist}\left(G_{r}\right)$ is symmetric.

Proof. According to [35, (I.9.7)], the smooth group scheme $G$ acts on the space of right integrals of $\operatorname{Dist}\left(G_{r}\right)$ via the character $g \mapsto \operatorname{det} \operatorname{Ad}(g)^{p^{r}-1}$. Since $G=(G, G) Z(G)$, cf. [31, (27.5)], this character is trivial, proving that the modular function of the Hopf algebra $\operatorname{Dist}\left(G_{r}\right)$ coincides with its counit. Consequently, $\operatorname{Dist}\left(G_{r}\right)$ is symmetric, see [23, (1.5)] for more details.

For future reference, we reformulate parts of [17, (7.1)].

Lemma 2.2. Let $\lambda \in X(T)$ be a weight. Then the following statements hold:

(1) $\operatorname{dim} \mathcal{V}_{G_{r}}\left(L_{r}(\lambda)\right) \neq 1$

(2) If $\operatorname{dim} \mathcal{V}_{G_{r}}\left(L_{r}(\lambda)\right)=2$, then the block $\mathcal{B} \subseteq \operatorname{Dist}\left(G_{r}\right)$ containing $L_{r}(\lambda)$ is Morita equivalent to a block of $\mathrm{SL}(2)_{r}$.

Proof. (1) This was shown in the second paragraph of [17, p.80].

(2) This follows directly from [17, p.80].

Theorem 2.3. Let $\lambda \in X(T)$ be a weight such that $L_{r}(\lambda)$ is not projective.

(1) If $\operatorname{dim} \mathcal{V}_{G_{r}}\left(L_{r}(\lambda)\right) \neq 2$, then $L_{r}(\lambda)$ is quasi-simple.

(2) If $\operatorname{dim} \mathcal{V}_{G_{r}}\left(L_{r}(\lambda)\right)=2$, then either $L_{r}(\lambda)$ is quasi-simple or $L_{r}(\lambda)$ belongs to a component of type $\mathbb{Z}\left[\tilde{A}_{12}\right]$. 
Proof. If $\operatorname{dim} \mathcal{V}_{G_{r}}\left(L_{r}(\lambda)\right) \geq 3$, then, thanks to [18, (2.2)], the component $\Theta$ containing $\left[L_{r}(\lambda)\right]$ is isomorphic to $\mathbb{Z}\left[A_{\infty}\right]$.

Assuming $\Theta \cong \mathbb{Z}\left[A_{\infty}\right]$, we show that the isoclass $\left[L_{r}(\lambda)\right]$ is located at an end of $\Theta$. Suppose that $L_{r}(\lambda)$ is not quasi-simple. Since $\operatorname{Dist}\left(G_{r}\right)$ is symmetric, [37, (1.5)] provides simple $\operatorname{Dist}\left(G_{r}\right)$ modules $L_{r}\left(\mu_{i}\right) \neq L_{r}(\lambda)$ for $1 \leq i \leq n$ such that each projective cover $P_{r}\left(\mu_{i}\right)$ is uniserial, of length $\ell\left(P_{r}\left(\mu_{i}\right)\right)=n+2$, with $\operatorname{Top}\left(\operatorname{Rad}\left(P_{r}\left(\mu_{i}\right)\right)\right) \cong L_{r}\left(\mu_{i-1}\right)$, where $\mu_{0}=\lambda$. By the same token, $L_{r}(\lambda)$ has multiplicity 1 in $P_{r}\left(\mu_{i}\right)$ for each $i$.

Assuming $n \geq 2$, we thus have

$$
\operatorname{dim}_{k} \operatorname{Ext}_{G_{r}}^{1}\left(L_{r}\left(\mu_{1}\right), L_{r}\left(\mu_{2}\right)\right)=0 \quad \text { and } \quad \operatorname{dim}_{k} \operatorname{Ext}_{G_{r}}^{1}\left(L_{r}\left(\mu_{2}\right), L_{r}\left(\mu_{1}\right)\right)=1,
$$

which contradicts [35, (II.9.19(2))]. Consequently, $n=1$, so that $\ell\left(P_{r}\left(\mu_{1}\right)\right)=3$. Owing to 35, (II.11.4)], the module $P_{r}\left(\mu_{1}\right)$ has a filtration by baby Verma modules. Since $\ell\left(P_{r}\left(\mu_{1}\right)\right)=3$, one $Z_{r}$-filtration factor, $Z_{r}(\gamma)$ say, is simple or projective. In view of [35, (II.11.8)], the factor $Z_{r}(\gamma)$ is simple and projective, so that the block containing $L_{r}(\lambda)$ is simple and $\operatorname{dim} \mathcal{V}_{G_{r}}\left(L_{r}(\lambda)\right)=0$, a contradiction.

By Lemma 2.2, the variety $\mathcal{V}_{G_{r}}\left(L_{r}(\lambda)\right)$ is not one-dimensional and so the proof of part (1) is complete.

Suppose that $\operatorname{dim} \mathcal{V}_{G_{r}}\left(L_{r}(\lambda)\right)=2$ and that $\Theta \neq \mathbb{Z}\left[A_{\infty}\right]$. By Theorem 4.1 in [18] and [21], the component containing $\left[L_{r}(\lambda)\right]$ is of type $\mathbb{Z}\left[\tilde{A}_{12}\right]$.

Remark. For $r=1$, a simple $G_{r}$-module of complexity 2 belongs to a component of type $\mathbb{Z}\left[\tilde{A}_{12}\right]$, cf. [17, (5.2)]. By contrast, simple modules of complexity 2 may belong to components of type $\mathbb{Z}\left[A_{\infty}\right]$, whenever $r \geq 2$ (cf. [21]).

We record an immediate consequence concerning the structure of the hearts of the principal indecomposable modules:

Corollary 2.4. Let $\lambda \in X(T)$ be a weight such that $L_{r}(\lambda)$ is not projective.

(1) If $\operatorname{dim} \mathcal{V}_{G_{r}}\left(L_{r}(\lambda)\right) \neq 2$, then $\mathrm{Ht}_{r}(\lambda)$ is indecomposable.

(2) If $\operatorname{dim} \mathcal{V}_{G_{r}}\left(L_{r}(\lambda)\right)=2$, then either $\operatorname{Ht}_{r}(\lambda)$ is indecomposable or $\operatorname{Ht}_{r}(\lambda) \cong L_{r}(\mu) \oplus L_{r}(\mu)$, where $L_{r}(\mu) \nRightarrow L_{r}(\lambda)$.

Proof. Owing to Lemma 2.1, the algebra $\operatorname{Dist}\left(G_{r}\right)$ is symmetric, so that $\operatorname{Soc}\left(P_{r}(\lambda)\right) \cong L_{r}(\lambda)$. Hence

$$
(0) \longrightarrow \operatorname{Rad}\left(P_{r}(\lambda)\right) \longrightarrow P_{r}(\lambda) \oplus \mathrm{Ht}_{r}(\lambda) \longrightarrow P_{r}(\lambda) / L_{r}(\lambda) \longrightarrow(0)
$$

is the standard almost split sequence originating in $\operatorname{Rad}\left(P_{r}(\lambda)\right)$, see [2, (V.5.5)]. Since $\Omega_{G_{r}}$ is an auto-equivalence of the stable module category of $\operatorname{Dist}\left(G_{r}\right),[2$, (IV.3.5)], it induces an automorphism of $\Gamma_{s}\left(G_{r}\right)$, [2, (X.1.9)]. Consequently, $L_{r}(\lambda)$ and $P_{r}(\lambda) / L_{r}(\lambda) \cong \Omega_{G_{r}}^{-1}\left(L_{r}(\lambda)\right)$ have the same number of non-projective predecessors. If the component $\Theta$ containing $L_{r}(\lambda)$ is isomorphic to $\mathbb{Z}\left[A_{\infty}\right]$, then the result thus follows from Theorem 2.3. Alternatively, $\operatorname{dim} \mathcal{V}_{G_{r}}\left(L_{r}(\lambda)\right)=2$ and $L_{r}(\lambda)$ belongs to a component of type $\mathbb{Z}\left[\tilde{A}_{12}\right]$. Thus, [13, (IV.3.8.3)] implies that $\operatorname{Ht}_{r}(\lambda) \cong L_{r}(\mu) \oplus L_{r}(\mu)$. The assumption $L_{r}(\lambda) \cong L_{r}(\mu)$ yields $\operatorname{dim}_{k} \operatorname{Ext}_{G_{r}}^{1}\left(L_{r}(\lambda), L_{r}(\lambda)\right)=2$. However, by Lemma 2.2, the block containing $L_{r}(\lambda)$ is Morita equivalent to a block of $\operatorname{Dist}\left(\operatorname{SL}(2)_{r}\right)$, and the simple modules of the latter algebra are known to afford no non-trivial self-extensions (cf. [43, Thm.]). 
Remark. The exceptional case of decomposable hearts corresponds to those blocks $\mathcal{B}_{r}(\lambda) \subseteq \operatorname{Dist}\left(G_{r}\right)$ that have tame representation type. The reader is referred to [13, (I.4)] for background information on representation type.

Suppose that $\mathrm{Ht}_{r}(\lambda)$ is decomposable. In light of [13, (IV.3.8.3)] and (2) of Corollary [2.4, the algebra $\mathcal{B}_{r}(\lambda) / \operatorname{Soc}\left(\mathcal{B}_{r}(\lambda)\right)$ is special biserial, and hence in particular tame or representation-finite (cf. [13, (II.3.1)]). Since $\mathcal{B}_{r}(\lambda)$ and $\mathcal{B}_{r}(\lambda) / \operatorname{Soc}\left(\mathcal{B}_{r}(\lambda)\right)$ have the same non-projective indecomposable modules, we conclude that $\mathcal{B}_{r}(\lambda)$ enjoys the same property. As $\operatorname{dim} \mathcal{V}_{G_{r}}\left(L_{r}(\lambda)\right)=2$, it follows that $\mathcal{B}_{r}(\lambda)$ is tame. Conversely, if $L_{r}(\lambda)$ belongs to a tame block $\mathcal{B}_{r}(\lambda)$ of $\operatorname{Dist}\left(G_{r}\right)$, then $\mathcal{B}_{r}(\lambda)$ is Morita equivalent to a tame block of $\operatorname{Dist}\left(\mathrm{SL}(2)_{1}\right)$, [17, (7.1)]. Consequently, the component containing $L_{r}(\lambda)$ is isomorphic to $\mathbb{Z}\left[\tilde{A}_{12}\right]$ and $\operatorname{Ht}_{r}(\lambda)$ is decomposable.

The final result of this section shows that, provided $G$ is defined over $\mathbb{F}_{p}$, every AR-component contains at most one simple module. The technical hypothesis on the derived subgroup $(G, G)$ of $G$ will later be removed by means of covering techniques, see Theorem 3.6. Since $G$ is defined over $\mathbb{F}_{p}$, we have the Frobenius endomorphism $F: G \longrightarrow G$, and for every $G$-module $M$, there is the Frobenius twist $M^{[1]}$, obtained by composing the representation afforded by $M$ with $F$ (see 35 , (II.3.16)]). For future reference, we record the following:

Lemma 2.5. Let $\Theta \subseteq \Gamma_{s}\left(G_{r}\right)$ be a component which is isomorphic to $\mathbb{Z}\left[\tilde{A}_{12}\right]$. Then $\Theta$ contains exactly one simple module.

Proof. According to [55, Thm.A] the component $\Omega_{G_{r}}^{-1}(\Theta) \cong \Theta$ is attached to a principal indecomposable module. In view of $\left[2\right.$, (V.5.5)] and Lemma 2.1, there thus exists a simple $G_{r}$-module $S$ such that $\left[\Omega_{G_{r}}^{-1}(S)\right] \in \Omega_{G_{r}}^{-1}(\Theta)$. As a result, $[S] \in \Theta$.

Corollary [2.4 in conjunction with [13, (IV.3.8.3)] implies that the block of $\operatorname{Dist}\left(G_{r}\right)$ containing $S$ possesses two simple modules $S$ and $T$, with

$$
(0) \longrightarrow \operatorname{Rad}(P(S)) \longrightarrow P(S) \oplus T \oplus T \longrightarrow P(S) / S \longrightarrow(0)
$$

being the almost split sequence involving the projective cover $P(S)$ of $S$. In particular, $T$ belongs to the component $\Omega_{G_{r}}^{-1}(\Theta)$. By virtue of [13, (IV.3.8.3)], we have $\Theta \neq \Omega_{G_{r}}(\Theta)$, so that $S$ is the only simple module belonging to $\Theta$.

Theorem 2.6. Suppose that $G$ is defined over $\mathbb{F}_{p}$ and that the derived subgroup $(G, G)$ of $G$ is simply connected. If $S$ is a simple, non-projective $G_{r}$-module, then $[S]$ is the only simple vertex in its stable AR-component.

Proof. Let $\Theta \subseteq \Gamma_{s}\left(G_{r}\right)$ be the stable AR-component containing the vertex $[S]$. According to Theorem 2.3 , the component $\Theta$ is of type $\mathbb{Z}\left[A_{\infty}\right]$ or $\mathbb{Z}\left[\tilde{A}_{12}\right]$. In the latter case, the assertion follows from Lemma 2.5.

Assuming $\Theta \cong \mathbb{Z}\left[A_{\infty}\right]$, we proceed by induction on $r$. If $r=1$, then [16, (4.1)] proves our claim. Now assume that $r \geq 2$ and let $T \neq S$ be another simple $G_{r}$-module which belongs to $\Theta$. According to Theorem 2.3. $S$ and $T$ lie at the end of $\Theta$. Thanks to Lemma 2.1, the Auslander-Reiten translate of $\Gamma_{s}\left(G_{r}\right)$ coincides with $\Omega_{G_{r}}^{2}$. Without loss of generality, there thus exists $n \in \mathbb{N}$ with

$$
T \cong \Omega_{G_{r}}^{2 n}(S) .
$$

As $(G, G)$ is simply connected, each element $\lambda \in X(T)$ can be written as $\lambda=\lambda_{0}+p^{r} \lambda_{1}$, with $\lambda_{0} \in X_{r}(T)$ and $\lambda_{1} \in X(T)$ (cf. [35, (II.9.14)]). Consequently, $X_{r}(T)$ contains a complete set of representatives for $X(T) / p^{r} X(T)$, and there exist $\lambda, \mu \in X_{r}(T)$ such that $S=L_{r}(\lambda), T=L_{r}(\mu)$, with both modules being restrictions of simple $G$-modules, see [35, (II.3.15)]. By the same token, 
there are decompositions $\mu=\mu_{0}+p \mu_{1}$ and $\lambda=\lambda_{0}+p \lambda_{1}$, where $\mu_{0}, \lambda_{0} \in X_{1}(T)$ and $\mu_{1}, \lambda_{1} \in$ $X_{r-1}(T)$. In view of [35, (II.3.16)], we thus have

$$
L_{r}(\mu) \cong L_{1}\left(\mu_{0}\right) \otimes_{k} L_{r-1}\left(\mu_{1}\right)^{[1]} \text { and } L_{r}(\lambda) \cong L_{1}\left(\lambda_{0}\right) \otimes_{k} L_{r-1}\left(\lambda_{1}\right)^{[1]}
$$

so that

$$
\left.L_{r}(\mu)\right|_{G_{1}}=m_{\mu} L_{1}\left(\mu_{0}\right) \text { and }\left.L_{r}(\lambda)\right|_{G_{1}}=m_{\lambda} L_{1}\left(\lambda_{0}\right) .
$$

Identity (2.7) in conjunction with standard properties of the Heller operator now yields:

$$
m_{\mu} L_{1}\left(\mu_{0}\right) \cong m_{\lambda} \Omega_{G_{1}}^{2 n}\left(L_{1}\left(\lambda_{0}\right)\right) \oplus \text { (proj.). }
$$

In particular, the indecomposable $G_{1}$-module $\Omega_{G_{1}}^{2 n}\left(L_{1}\left(\lambda_{0}\right)\right.$ ) (cf. [29]) is a direct summand of $L_{1}\left(\mu_{0}\right)$. Consequently, [3, (2.5.4)] gives

$$
\operatorname{dim}_{k} \operatorname{Ext}_{G_{1}}^{2 n}\left(L_{1}\left(\lambda_{0}\right), L_{1}\left(\lambda_{0}\right)\right) \leq \operatorname{dim}_{k} \operatorname{Hom}_{G_{1}}\left(L_{1}\left(\mu_{0}\right), L_{1}\left(\lambda_{0}\right)\right) \leq 1 .
$$

Owing to [15, (2.1)], this implies $\mathrm{cx}_{G_{1}}\left(L_{1}\left(\lambda_{0}\right)\right) \leq 1$, which, in view of $G$ being reductive, entails the projectivity of $L_{1}\left(\lambda_{0}\right)$, see [16, (3.1)]. By (2.8), the simple $G_{1}$-module $L_{1}\left(\mu_{0}\right)$ also has this property.

Let $e_{S}, e_{T} \in \operatorname{Dist}\left(G_{1}\right)$ be the primitive central idempotents defining the simple blocks containing $\left.S\right|_{G_{1}}$ and $\left.T\right|_{G_{1}}$, respectively. Since these are fixed by the adjoint representation of the connected group $G$, it follows that $e_{S}, e_{T}$ are also central idempotents of $\operatorname{Dist}\left(G_{r}\right) \supseteq \operatorname{Dist}\left(G_{1}\right)$ (see 35 , (II.10.3)] for more details). As $S$ and $T$ belong to the same block of $\operatorname{Dist}\left(G_{r}\right)$, the idempotents $e_{S}$ and $e_{T}$ act via the identity on $S$ and $T$. Consequently, $e_{S}=e_{T}$, so that the simple projective $G_{1}$-modules $L_{1}\left(\lambda_{0}\right)$ and $L_{1}\left(\mu_{0}\right)$ belong to the same block, whence $\operatorname{Hom}_{G_{1}}\left(L_{1}\left(\lambda_{0}\right), L_{1}\left(\mu_{0}\right)\right)=k$. Let $\gamma \in X(G)$ be the character giving the action of $G$ on $\operatorname{Hom}_{G_{1}}\left(L_{1}\left(\lambda_{0}\right), L_{1}\left(\mu_{0}\right)\right)$. There results an isomorphism

of $G_{r}$-modules. As a result, we have

$$
L_{1}\left(\mu_{0}\right) \cong L_{1}\left(\lambda_{0}\right) \otimes_{k} k_{\gamma}
$$

$$
T \cong L_{1}\left(\lambda_{0}\right) \otimes_{k} L_{r-1}\left(\mu_{1}\right)^{[1]} \otimes_{k} k_{-\gamma} .
$$

Since $G_{1} \subseteq \operatorname{ker} \gamma$, it follows that $\gamma \in p X(G)$ (cf. [35, (II.1.18,II.3.7)]), so that the last two tensor factors are the Frobenius twist of $L_{r-1}\left(\mu_{1}\right) \otimes_{k} k_{\omega}$, for some $\omega \in X(G)$.

Owing to [35, (II.10.5)], the functor

$$
M \mapsto L_{1}\left(\lambda_{0}\right) \otimes_{k} M^{[1]}
$$

induces an equivalence between $\bmod G_{r-1}$ and the sum of those blocks of $\bmod G_{r}$ given by $e_{S}$. As a result, the modules $L_{r-1}\left(\mu_{1}\right) \otimes_{k} k_{\omega}$ and $L_{r-1}\left(\lambda_{1}\right)$ belong to the same component of $\Gamma_{s}\left(G_{r-1}\right)$. The inductive hypothesis now provides an isomorphism $L_{r-1}\left(\mu_{1}\right) \otimes_{k} k_{\omega} \cong L_{r-1}\left(\lambda_{1}\right)$ of $G_{r-1}$-modules, so that

a contradiction.

$$
T \cong L_{1}\left(\lambda_{0}\right) \otimes_{k}\left(L_{r-1}\left(\mu_{1}\right) \otimes_{k} k_{\omega}\right)^{[1]} \cong L_{1}\left(\lambda_{0}\right) \otimes_{k} L_{r-1}\left(\lambda_{1}\right)^{[1]} \cong S,
$$

\section{Auslander-Reiten Theory for $\bmod G_{r} T$}

Let $G$ be a connected, reductive, smooth group scheme with maximal torus $T$ and character group $X(T)$. Given $\lambda \in X(T)$, we denote by $\Theta_{r}(\lambda)$ the connected component of the stable AuslanderReiten quiver $\Gamma_{s}\left(G_{r}\right)$ of Dist $\left(G_{r}\right)$ containing the isoclass $\left[Z_{r}(\lambda)\right]$. Our main tool in this section is covering theory, appearing in the guise of Jantzen's category $\bmod G_{r} T$ of $G_{r} T$-modules (cf. 32, $\S 2])$. It turns out that certain properties of $\Theta_{r}(\lambda)$ can be effectively studied by first investigating the corresponding properties for components of the stable Auslander-Reiten quiver of the Frobenius category $\bmod G_{r} T$. In view of the conjugacy of maximal tori, our considerations do not depend on the choice of $T$. The category $\bmod G_{r} T$ is a highest weight category in the sense of [10], whose 
standard objects $\Delta(\lambda)$ are the baby Verma modules $\widehat{Z}_{r}(\lambda)$, where $\lambda \in X(T)$. Recall that the $G_{r} T$-modules $\widehat{Z}_{r}(\lambda)$ and $\widehat{Z}_{r}^{\prime}(\lambda)$ are given by

$$
\widehat{Z}_{r}(\lambda):=\operatorname{Dist}\left(G_{r}\right) \otimes_{\operatorname{Dist}\left(B_{r}\right)} k_{\lambda} \text { and } \widehat{Z}_{r}^{\prime}(\lambda):=\operatorname{Hom}_{\operatorname{Dist}\left(B_{r}^{-}\right)}\left(\operatorname{Dist}\left(G_{r}\right), k_{\lambda}\right),
$$

where $B^{-}$is the Borel subalgebra opposite to our standard Borel subalgebra $B \subseteq G$. The $T$-action is induced by the adjoint action of $T$ on $\operatorname{Dist}\left(G_{r}\right)$ (cf. [35, §II.9]).

We shall consider the full subcategory $\mathcal{F}(\Delta)$ of $\bmod G_{r} T$ of $\widehat{Z}_{r}$-filtered $G_{r} T$-modules. Given $M \in \mathcal{F}(\Delta)$, its filtration multiplicities

$$
\left[M: \widehat{Z}_{r}(\lambda)\right]
$$

are well-defined (cf. [35, (II.11.2)]). We define the $\Delta$-support of $M \in \mathcal{F}(\Delta)$ via

$$
\operatorname{supp}_{\Delta}(M):=\left\{\lambda \in X(T) \mid\left[M: \widehat{Z}_{r}(\lambda)\right] \neq 0\right\} .
$$

The objects of $\mathcal{F}(\Delta)$ are usually referred to as $\Delta$-good modules.

Given $\lambda \in X(T)$, we write $X(T)_{\geq \lambda}:=\{\mu \in X(T) \mid \mu \geq \lambda\}$ as well as $X(T)_{>\lambda}:=\{\mu \in X(T) \mid \mu>$ $\lambda\}$.

Recall that for every $\lambda \in X(T)$ there exists a unique simple object $\widehat{L}_{r}(\lambda)$ with projective cover $\widehat{P}_{r}(\lambda)$, and that all simple and projective indecomposable objects are of this form (see [35, (II.9.6), (II.11.5.(3))]). In view of [35, (II.11.4)], the module $\widehat{P}_{r}(\lambda)$ belongs to $\mathcal{F}(\Delta)$, with its filtration multiplicities obeying the BGG reciprocity formula

$$
\left[\widehat{P}_{r}(\lambda): \widehat{Z}_{r}(\mu)\right]=\left[\widehat{Z}_{r}(\mu): \widehat{L}_{r}(\lambda)\right]
$$

In particular, the highest weights of the $\widehat{Z}_{r}$-filtration factors of $\widehat{P}_{r}(\lambda)$ enjoy the following property:

Lemma 3.1. We have

$$
\operatorname{supp}_{\Delta}\left(\widehat{P}_{r}(\lambda)\right) \subseteq X(T)_{\geq \lambda}
$$

for every $\lambda \in X(T)$.

In the sequel, we shall study $\bmod G_{r} T$ as well as $\bmod \left(G_{r} \rtimes T\right)$. The latter category coincides with the category $\bmod _{X(T)} \operatorname{Dist}\left(G_{r}\right)$ of $X(T)$-graded $G_{r}$-modules and degree zero homomorphisms. We identify $X(T)$ with the subgroup of those characters $\lambda \in X\left(G_{r} \rtimes T\right)$ that are trivial on $G_{r}$. Thus, every $\lambda \in X(T)$ defines a one-dimensional $\left(G_{r} \rtimes T\right)$-module $k_{\lambda}$. It now follows directly from the definition that the shift functor $M \mapsto M\langle\lambda\rangle$ of $\bmod _{X(T)} \operatorname{Dist}\left(G_{r}\right)$ corresponds to the auto-equivalence $M \mapsto M \otimes_{k} k_{\lambda}$.

Since $G_{r} T \cong\left(G_{r} \rtimes T\right) / T_{r}$, the shifts sending $\bmod G_{r} T$ onto itself are given by those $\lambda \in X(T)$ that vanish on $T_{r}$. In view of [35, (II.3.7)] there exists an exact sequence

$$
(0) \longrightarrow p^{r} X(T) \stackrel{\text { can }}{\longrightarrow} X(T) \stackrel{\text { res. }}{\longrightarrow} X\left(T_{r}\right) \longrightarrow(0),
$$

so that the relevant shifts are those belonging to the subgroup $p^{r} X(T)$ of $X(T)$.

The block decomposition of the algebraic group $G_{r} \rtimes T$ given in [35, (II.7.1)] yields a direct sum decomposition

$$
\bmod \left(G_{r} \rtimes T\right)=\bigoplus_{b \in \mathcal{B}\left(G_{r} \rtimes T\right)}\left(\bmod \left(G_{r} \rtimes T\right)\right)_{b},
$$

whose constituents are referred to as blocks. The proof of [19, (2.1)] for $r=1$ may be adopted verbatim to obtain: 
Lemma 3.2. The following statements hold:

(1) The category $\bmod G_{r} T$ is a sum of blocks of $\bmod \left(G_{r} \rtimes T\right)$.

(2) The category $\bmod G_{r} T$ has almost split sequences.

(3) The canonical restriction functor $\mathfrak{F}: \bmod G_{r} T \longrightarrow \bmod G_{r}$ sends indecomposables to indecomposables and almost split sequences to almost split sequences.

Recall from [35, (II.9.4)] that an object $P \in \bmod G_{r} T$ is projective if and only if it is injective. Thus, $\bmod G_{r} T$ is a Frobenius category (cf. [28, (I.2)]), and we can speak of the stable Auslander-Reiten quiver $\Gamma_{s}\left(G_{r} T\right)$ of $G_{r} T$. In view of Lemma $3.2(3)$, the functor $\mathfrak{F}$ commutes with the AuslanderReiten translations $\tau_{G_{r} T}$ and $\tau_{G_{r}}$ of $\Gamma_{s}\left(G_{r} T\right)$ and $\Gamma_{s}\left(G_{r}\right)$, that is,

$$
\tau_{G_{r}} \circ \mathfrak{F}=\mathfrak{F} \circ \tau_{G_{r} T} .
$$

We record the analogue of [19, (2.2)]:

Lemma 3.3. The following statements hold:

(1) The canonical restriction functor $\mathfrak{F}: \bmod G_{r} T \longrightarrow \bmod G_{r}$ induces a morphism $\mathfrak{F}$ : $\Gamma_{s}\left(G_{r} T\right) \longrightarrow \Gamma_{s}\left(G_{r}\right)$ of stable translation quivers that maps the set $x^{+}$of successors of an arbitrary vertex $x \in \Gamma_{s}\left(G_{r} T\right)$ onto the set of successors $\mathfrak{F}(x)^{+}$of $\mathfrak{F}(x)$.

(2) If $\Theta \subseteq \Gamma_{s}\left(G_{r} T\right)$ is a component, then $\mathfrak{F}(\Theta)$ is a component of $\Gamma_{s}\left(G_{r}\right)$.

By work of Gordon and Green [27, $§ 1, \S 3]$, the Auslander-Reiten translation $\tau_{G_{r} T}=\left.\tau_{G_{r} \rtimes T}\right|_{\bmod G_{r} T}$ is given by

$$
\tau_{G_{r} T}(M)=\operatorname{Tr}(M)^{*}
$$

where $\operatorname{Tr}(M)$ denotes the transpose of $M$ (see [2, (IV.1)] for the definition). In view of $\bmod G_{r} T$ being Frobenius, we have natural isomorphisms

$$
\operatorname{Tr}(M)^{*} \cong \mathcal{N}\left(\Omega_{G_{r} T}^{2}(M)\right),
$$

where $\mathcal{N}=\operatorname{Hom}_{G_{r}}\left(-, \operatorname{Dist}\left(G_{r}\right)\right)^{*}$ is the Nakayama functor of $\bmod G_{r} T$. According to [35, (I.9.7)], the group $G$ acts via the character $g \mapsto \operatorname{det}(\operatorname{Ad}(g))^{p^{r}-1}$ on the space of left integrals of $\operatorname{Dist}\left(G_{r}\right)$. The reductivity of $G$ implies that this character is trivial, and we obtain $\mathcal{N} \cong \operatorname{id}_{\bmod G_{r} T}$, cf. 35, (I.8.12)] and [21]. As a result, we have

$$
\tau_{G_{r} T}(M) \cong \Omega_{G_{r} T}^{2}(M)
$$

for every $G_{r} T$-module $M$.

Replacing $U_{0}(\mathfrak{g})$ by $\operatorname{Dist}\left(G_{r}\right)$ in [19, (2.3)] while observing [18, (1.3)], one obtains:

Proposition 3.4. Let $\Theta \subseteq \Gamma_{s}\left(G_{r} T\right)$ be a component. Then the tree class $\bar{T}_{\Theta}$ of $\Theta$ is a simply laced finite or infinite Dynkin diagram, a simply laced Euclidean diagram, or $\tilde{A}_{12}$.

In contrast to $\bmod G_{r}$, the category $\bmod G_{r} T$ behaves well under passage to coverings. Given a connected smooth reductive group scheme $G$, a connected smooth reductive group scheme $\tilde{G}$ is called a covering of $G$ if there exists a finite diagonalizable subgroup scheme $\tilde{Z} \subseteq \tilde{G}$ such that $\tilde{G} / \tilde{Z} \cong G$ (cf. [35, (II.1.17)]).

Lemma 3.5. Let $\tilde{G}$ be a covering of $G$ with maximal torus $\tilde{T} \subseteq \tilde{G}$ such that $\tilde{T} / \tilde{Z} \cong T$. Then $\bmod G_{r} T$ is the sum of those blocks of $\bmod \tilde{G}_{r} \tilde{T}$ whose characters belong to $X(T) \subseteq X(\tilde{T})$. 
Proof. Owing to [35, (II.9.7)], we have $\left(\tilde{G}_{r} \tilde{T}\right) / \tilde{Z} \cong G_{r} T$. By rigidity of tori (cf. [53, (7.7)]), the group $\tilde{Z}$ belongs to the center of $\tilde{G}$. Thus, $\tilde{Z}$ acts on each module of a block of $\bmod \tilde{G}_{r} \tilde{T}$ via the same character (cf. [35, (II.7.1)]), and our assertion follows.

Recall that an indecomposable module $M$ of a self-injective algebra $\Lambda$ is periodic, if there exists $m \in \mathbb{N}$ such that $\Omega_{\Lambda}^{m}(M) \cong M$. Periodic modules have complexity 1 . It thus follows from Lemma 2.2 that the simple $G_{r}$-modules $L_{r}(\lambda)$ are not periodic.

The foregoing result enables us to generalize Theorem 2.6 to arbitrary reductive groups:

Theorem 3.6. Suppose that $G$ is defined over $\mathbb{F}_{p}$ and let $\lambda \in X(T)$.

(1) If the component $\Theta \subseteq \Gamma_{s}\left(G_{r}\right)$ containing $L_{r}(\lambda)$ is isomorphic to $\mathbb{Z}\left[A_{\infty}\right]$, then $\widehat{L}_{r}(\lambda)$ is the only simple vertex in its AR-component.

(2) The module $L_{r}(\lambda)$ is the only simple vertex in its AR-component.

Proof. (1) General theory ensures the existence of a covering $\tilde{G}$ such that $(\tilde{G}, \tilde{G})$ is simply connected. In view of Lemma 3.5, we may thus assume without loss of generality that the derived group $(G, G)$ of $G$ is simply connected.

Suppose that $\widehat{L}_{r}\left(\lambda^{\prime}\right)$ also belongs to the component $\widehat{\Theta}$ of $\Gamma_{s}\left(G_{r} T\right)$ containing $\widehat{L}_{r}(\lambda)$. Thanks to Lemma 3.3. the component $\Theta=\mathfrak{F}(\widehat{\Theta})$ of $\Gamma_{s}\left(G_{r}\right)$ contains $L_{r}(\lambda)$ and $L_{r}\left(\lambda^{\prime}\right)$, and a consecutive application of Theorem [2.6 and [35, (II.3.10)] implies

$$
\lambda^{\prime}=\lambda+p^{r} \gamma
$$

for some $\gamma \in X(T)$.

In light of Lemma 3.3 and our current assumption, the component $\widehat{\Theta}$ has infinitely many $\tau_{G_{r} T^{-}}$ orbits, and Proposition 3.4 shows that its tree class $\bar{T}_{\widehat{\Theta}}$ is isomorphic to $A_{\infty}, A_{\infty}^{\infty}$, or $D_{\infty}$. Since the number of non-projective direct summands of the middle terms of almost split sequences in $\widehat{\Theta}$ takes the values 1 and 2, the latter two alternatives cannot occur.

As $L_{r}(\lambda)$ is quasi-simple, it now follows from Lemma 3.2 that $\widehat{L}_{r}(\lambda)$ and $\widehat{L}_{r}\left(\lambda^{\prime}\right)$ both lie at ends of $\widehat{\Theta}$. Consequently, there exists $m \in \mathbb{Z}$ with

$$
\Omega_{G_{r} T}^{2 m}\left(\widehat{L}_{r}(\lambda)\right) \cong \widehat{L}_{r}\left(\lambda^{\prime}\right) .
$$

Application of $\mathfrak{F}$ gives

$$
\Omega_{G_{r}}^{2 m}\left(L_{r}(\lambda)\right) \cong L_{r}\left(\lambda^{\prime}\right) \cong L_{r}(\lambda) .
$$

Since the simple $G_{r}$-modules are not periodic, we conclude $m=0$ and $\lambda=\lambda^{\prime}$.

(2) Let $\Theta \subseteq \Gamma_{s}\left(G_{r}\right)$ be the component containing $L_{r}(\lambda)$. Thanks to Theorem 2.3, we have $\Theta \cong \mathbb{Z}\left[A_{\infty}\right], \mathbb{Z}\left[\tilde{A}_{12}\right]$. In the latter case, our assertion follows from Lemma 2.5.

Suppose that $\Theta \cong \mathbb{Z}\left[A_{\infty}\right]$ and denote by $\widehat{\Theta}$ the stable AR-component of $\widehat{L}_{r}(\lambda)$. The assumption $\left[L_{r}(\mu)\right] \in \Theta=\mathfrak{F}(\widehat{\Theta})$ implies the existence of $\gamma \in X(T)$ with $\left[\widehat{L}_{r}\left(\mu+p^{r} \gamma\right)\right] \in \widehat{\Theta}$ (see [35, (II.3.10)]). According to (1), we have $\lambda=\mu+p^{r} \gamma$, whence $L_{r}(\lambda) \cong L_{r}(\mu)$, as desired.

Remark. If $\Theta \cong \mathbb{Z}\left[\tilde{A}_{12}\right]$, then the AR-component containing $\widehat{L}_{r}(\lambda)$ may have several simple vertices, see [19, p.749f].

We turn to the consideration of $\Delta$-good modules of the highest weight category $\bmod G_{r} T$. Using the rank varieties $V_{r}(G)_{M}$, the arguments employed in [19, (4.1)] imply: 
Theorem 3.7. Let $M$ be an indecomposable $G_{r} T$-module, $\Theta \subseteq \Gamma_{s}\left(G_{r} T\right)$ and $\Psi \subseteq \Gamma_{s}\left(G_{r}\right)$ be the stable AR-components containing $[M]$ and $[\mathfrak{F}(M)]$, respectively.

(1) Every vertex of $\Psi$ has a $G_{r} T$-structure.

(2) If $M$ affords a $\widehat{Z}_{r}$-filtration, so does every indecomposable $G_{r} T$-module belonging to $\Theta$.

(3) If $\mathfrak{F}(M)$ affords a $Z_{r}$-filtration, so does every indecomposable $G_{r}$-module belonging to $\Psi$.

We conclude this section by recording two elementary, but useful observations:

Lemma 3.8. Let

$$
(0) \longrightarrow M^{\prime} \longrightarrow M \longrightarrow M^{\prime \prime} \longrightarrow(0)
$$

be an exact sequence of $G_{r} T$-modules. If two modules of the sequence belong to $\mathcal{F}(\Delta)$, so does the third. In that case, we have

$$
\left[M: \widehat{Z}_{r}(\lambda)\right]=\left[M^{\prime}: \widehat{Z}_{r}(\lambda)\right]+\left[M^{\prime \prime}: \widehat{Z}_{r}(\lambda)\right]
$$

for every $\lambda \in X(T)$.

Proof. Recall that $\bmod B_{r}^{-}$is a Frobenius category. If two modules of the above sequence are $B_{r}^{-}$injective, so is the third. Thus, the first assertion follows directly from [35, (II.11.2)]. By the same token, we have

$$
\left[M: \widehat{Z}_{r}(\lambda)\right]=\operatorname{dim}_{k} \operatorname{Hom}_{G_{r} T}\left(M, \widehat{Z}_{r}^{\prime}(\lambda)\right),
$$

so that the additivity of the filtration multiplicities follows by applying $\operatorname{Hom}_{G_{r} T}\left(-, \widehat{Z}_{r}^{\prime}(\lambda)\right.$, while observing $\left.\operatorname{Ext}_{G_{r} T}^{1}\left(\widehat{Z}_{r}(\mu)\right), \widehat{Z}_{r}^{\prime}(\lambda)\right)=(0)$ for all $\mu \in X(T)$, see [35, (II.9.9)].

Lemma 3.9. Let $M \in \mathcal{F}(\Delta)$. Then $\operatorname{Top}_{G_{r} T}(M)$ is a direct summand of

$$
\bigoplus_{\lambda \in \operatorname{supp}_{\Delta}(M)}\left[M: \widehat{Z}_{r}(\lambda)\right] \widehat{L}_{r}(\lambda) .
$$

Proof. We use induction on the $\widehat{Z}_{r}$-filtration length $\ell_{\Delta}(M)$ of $M$, the case $\ell_{\Delta}(M)=1$ being a consequence of [35, (II.9.6(4))]. An exact sequence

$$
(0) \longrightarrow M^{\prime} \longrightarrow M \longrightarrow \widehat{Z}_{r}(\mu) \longrightarrow(0)
$$

of $G_{r} T$-modules induces an exact sequence

$$
\operatorname{Top}_{G_{r} T}\left(M^{\prime}\right) \longrightarrow \operatorname{Top}_{G_{r} T}(M) \longrightarrow \widehat{L}_{r}(\mu) \longrightarrow(0),
$$

so that $\operatorname{Top}_{G_{r} T}(M)$ is a direct summand of $\operatorname{Top}_{G_{r} T}\left(M^{\prime}\right) \oplus \widehat{L}_{r}(\mu)$. Lemma 3.8 yields

$$
\left[M: \widehat{Z}_{r}(\lambda)\right]=\left[M^{\prime}: \widehat{Z}_{r}(\lambda)\right]+\delta_{\lambda, \mu},
$$

and by inductive hypothesis, $\operatorname{Top}_{G_{r} T}(M)$ is a direct summand of

$$
\bigoplus_{\lambda \in \operatorname{supp}_{\Delta}\left(M^{\prime}\right)}\left[M^{\prime}: \widehat{Z}_{r}(\lambda)\right] \widehat{L}_{r}(\lambda) \oplus \widehat{L}_{r}(\mu)=\bigoplus_{\lambda \in \operatorname{supp}_{\Delta}(M)}\left[M: \widehat{Z}_{r}(\lambda)\right] \widehat{L}_{r}(\lambda),
$$

as desired. 


\section{Auslander-Reiten Components of Verma Modules}

Given a component $\Theta \subseteq \Gamma_{s}\left(G_{r}\right)$, we recall that any two vertices of $\Theta$ have the same rank variety $V_{r}(G)_{\Theta}\left(\right.$ cf. [18, (1.1)]). The quiver $\Gamma_{s}\left(G_{r}\right)$ is known to satisfy an analogue of Webb's Theorem [55] for finite groups, that is, the tree classes of the components of $\Gamma_{s}\left(G_{r}\right)$ are finite or infinite Dynkin diagrams, or Euclidean diagrams (cf. [18, (1.3)]). Our next lemma further reduces the possibilities for the components $\Theta_{r}(\lambda)$ containing the isoclasses $\left[Z_{r}(\lambda)\right]$.

The group $G$ acts on $G_{r}$ via conjugation. This action induces operations on $V_{r}(G)$ and $\operatorname{Dist}\left(G_{r}\right)$, where we denote the automorphism of $\operatorname{Dist}\left(G_{r}\right)$ corresponding to $g$ by $\operatorname{Ad}(g)$. Letting $M^{(g)}$ be the pull-back of a $G_{r}$-module $M$ along $\operatorname{Ad}(g)^{-1}$, we obtain actions of $G$ on $\bmod G_{r}$ and $\Gamma_{s}\left(G_{r}\right)$ via auto-equivalences and isomorphisms of stable representation quivers, respectively. Note that

$$
V_{r}(G)_{M^{(g)}}=g \cdot V_{r}(G)_{M} \quad \forall g \in G .
$$

Since $G$ is connected, the isoclasses of the simple $G_{r}$-modules are fixed by the above action, so that the rank varieties of these modules are $G$-stable subsets of $V_{r}(G)$.

A component of $\Gamma_{s}\left(G_{r}\right)$ is regular if it is not attached to a principal indecomposable module.

Lemma 4.1. Let $\lambda \in X(T)$ be a weight such that $Z_{r}(\lambda)$ is not projective. Then the following statements hold:

(1) The component $\Theta_{r}(\lambda)$ is regular.

(2) The isoclass $\left[Z_{r}(\lambda)\right]$ is a vertex of $\Theta_{r}(\lambda)$ of minimal dimension.

(3) If $\operatorname{dim} V_{r}(G)_{\Theta_{r}(\lambda)}=1$, then $\Theta_{r}(\lambda) \cong \mathbb{Z}\left[A_{\infty}\right] /\left(\tau^{p^{n}}\right)$ for some $n \in\{0, \ldots, r-1\}$.

(4) If $\operatorname{dim} V_{r}(G)_{\Theta_{r}(\lambda)} \geq 2$, then $\Theta_{r}(\lambda) \cong \mathbb{Z}\left[A_{\infty}\right], \mathbb{Z}\left[D_{\infty}\right]$, or $\mathbb{Z}\left[A_{\infty}^{\infty}\right]$.

Proof. Thanks to [50, $(1.5,1.14)]$ and [51, (5.2)], there is a commutative diagram

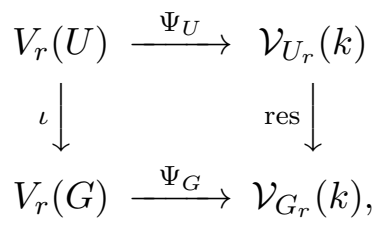

where $\iota$ is injective and the horizontal arrows are homeomorphisms. Owing to Proposition 1.2 we have $\mathcal{V}_{G_{r}}\left(Z_{r}(\lambda)\right) \subseteq$ im res. Thus, [51, (6.8)] gives $V_{r}(G)_{\Theta_{r}(\lambda)} \subseteq V_{r}(U)$.

Let $g_{0} \in G$ be a representative of the longest element $w_{0}$ of the Weyl group $W$ of $G$, so that $g_{0} U g_{0}^{-1}=U^{-}$, cf. [35, (II.1.8)]. For any non-trivial element $\varphi \in V_{r}(G)_{\Theta_{r}(\lambda)} \subseteq V_{r}(U)$, we have $g_{0} . \varphi \in V_{r}\left(U^{-}\right)$, so that $g_{0} \cdot \varphi \notin V_{r}(G)_{\Theta_{r}(\lambda)}$. Thus, $V_{r}(G)_{\Theta_{r}(\lambda)}$ is not $G$-invariant.

(1) Since rank varieties of non-regular components are necessarily $G$-invariant (cf. [17, $\S 7]$ ), assertion (1) follows.

(2) This is a direct consequence of Theorem 3.7 (3).

(3) A consecutive application of [18, (2.1)] and [18, (4.1)] shows that $\Theta_{r}(\lambda) \cong \mathbb{Z}\left[A_{\infty}\right] /\left(\tau^{m}\right)$ for some $m \in \mathbb{N}$. According to Lemma [2.1. Dist $\left(G_{r}\right)$ is a symmetric algebra, so that $\tau_{G_{r}}=\Omega_{G_{r}}^{2}$. The Friedlander-Suslin Theorem [25, (1.5)] shows that the even cohomology ring $\mathrm{H}^{\bullet}\left(G_{r}, k\right)$ is a finitely generated module of a graded subalgebra, whose generators have degree $2 p^{i}$ for $0 \leq i \leq r-1$. Thanks to [4, (5.10.6)], the periods of the periodic modules are divisors of these numbers. This implies our assertion concerning $n$.

(4) Suppose that assertion (4) does not hold. The classification of AR-components (cf. [18, (4.1)]) now implies that $\Theta_{r}(\lambda)$ is a Euclidean component. As such, it is not regular, contradicting part (1). 
Our next result implies that the vertex $\left[\widehat{Z}_{r}(\lambda)\right]$ has exactly one predecessor in $\Gamma_{s}\left(G_{r} T\right)$, thereby showing in particular that $\Theta_{r}(\lambda) \varsubsetneqq \mathbb{Z}\left[A_{\infty}^{\infty}\right]$.

Theorem 4.2. Suppose that $\widehat{Z}_{r}(\lambda)$ is not projective and let $\widehat{E}$ be the middle term of the almost split sequence

$$
(0) \longrightarrow \Omega_{G_{r} T}^{2}\left(\widehat{Z}_{r}(\lambda)\right) \longrightarrow \widehat{E} \longrightarrow \widehat{Z}_{r}(\lambda) \longrightarrow(0)
$$

Then the following statements hold:

(1) $\operatorname{supp}_{\Delta}(\widehat{E}) \subseteq X(T)_{\geq \lambda}$ and $\left[\widehat{E}: \widehat{Z}_{r}(\lambda)\right]=1$.

(2) The module $\widehat{E}$ is indecomposable.

Proof. We consider a minimal projective presentation

$$
(0) \longrightarrow \Omega_{G_{r} T}^{2}\left(\widehat{Z}_{r}(\lambda)\right) \longrightarrow \widehat{P}_{1} \longrightarrow \widehat{P}_{r}(\lambda) \longrightarrow \widehat{Z}_{r}(\lambda) \longrightarrow(0)
$$

of the $G_{r} T$-module $\widehat{Z}_{r}(\lambda)$. Since $\left[\widehat{Z}_{r}(\lambda): \widehat{L}_{r}(\lambda)\right]=1$ (cf. [35, (II.9.6)]), Lemma 3.1] in conjunction with Lemma 3.8 implies the inclusion $\operatorname{supp}_{\Delta}\left(\Omega_{G_{r} T}\left(\widehat{Z}_{r}(\lambda)\right) \subseteq X(T)_{>\lambda}\right.$. Owing to Lemma 3.9, the module $\operatorname{Top}_{G_{r} T}\left(\Omega_{G_{r} T}\left(\widehat{Z}_{r}(\lambda)\right)\right)$ is a direct summand of

$$
\bigoplus_{\mu \in \operatorname{supp}_{\Delta}\left(\Omega_{G_{r} T}\left(\widehat{Z}_{r}(\lambda)\right)\right)}\left[\Omega_{G_{r} T}\left(\widehat{Z}_{r}(\lambda)\right): \widehat{Z}_{r}(\mu)\right] \widehat{L}_{r}(\mu) .
$$

Another application of Lemma 3.1 therefore yields $\operatorname{supp}_{\Delta}\left(\widehat{P}_{1}\right) \subseteq X(T)_{>\lambda}$. In particular, the module $\widehat{Z}_{r}(\lambda)$ is not a filtration factor of $\Omega_{G_{r} T}^{2}\left(\widehat{Z}_{r}(\lambda)\right)$ and Lemma 3.8 implies

$$
\left[\widehat{E}: \widehat{Z}_{r}(\lambda)\right]=1 .
$$

In virtue of Theorem $3.7(2)$, every indecomposable constituent of $\widehat{E}$ is $\widehat{Z}_{r}$-filtered, so that each irreducible morphism terminating in $\widehat{Z}_{r}(\lambda)$ is surjective (see [2, (V.5.1)]). Thus, if $\widehat{E}_{i}$ is an indecomposable constituent of $\widehat{E}$, then, by [2, (V.5.3)], our almost split sequence induces an exact sequence

$$
(0) \longrightarrow X \longrightarrow \widehat{E}_{i} \longrightarrow \widehat{Z}_{r}(\lambda) \longrightarrow(0)
$$

and Lemma 3.8 gives rise to

$$
\left[\widehat{E}_{i}: \widehat{Z}_{r}(\lambda)\right]=\left[X: \widehat{Z}_{r}(\lambda)\right]+\left[\widehat{Z}_{r}(\lambda): \widehat{Z}_{r}(\lambda)\right]=\left[X: \widehat{Z}_{r}(\lambda)\right]+1
$$

Consequently, $\widehat{E}$ can only have one indecomposable constituent, so that (2) follows.

A twofold application of Lemma 3.8 yields

$$
\operatorname{supp}_{\Delta}(\widehat{E})=\operatorname{supp}_{\Delta}\left(\widehat{Z}_{r}(\lambda)\right) \cup \operatorname{supp}_{\Delta}\left(\Omega_{G_{r} T}^{2}\left(\widehat{Z}_{r}(\lambda)\right)\right) \subseteq \operatorname{supp}_{\Delta}\left(\widehat{Z}_{r}(\lambda)\right) \cup \operatorname{supp}_{\Delta}\left(\widehat{P}_{1}\right) \subseteq X(T)_{\geq \lambda},
$$

as desired.

Corollary 4.3. Suppose that $Z_{r}(\lambda)$ is not projective. The middle term $E$ of the almost split sequence

$$
(0) \longrightarrow \Omega_{G_{r}}^{2}\left(Z_{r}(\lambda)\right) \longrightarrow E \longrightarrow Z_{r}(\lambda) \longrightarrow(0)
$$

is indecomposable. 
Proof. According to [35, (II.9.4)] the $G_{r} T$-module $\widehat{Z}_{r}(\lambda)$ is not projective. We consider the almost split sequence

$$
(0) \longrightarrow \Omega_{G_{r} T}^{2}\left(\widehat{Z}_{r}(\lambda)\right) \longrightarrow \widehat{E} \longrightarrow \widehat{Z}_{r}(\lambda) \longrightarrow(0)
$$

terminating in $\widehat{Z}_{r}(\lambda)$. Since $Z_{r}(\lambda)=\mathfrak{F}\left(\widehat{Z}_{r}(\lambda)\right)$, Lemma $3.2(3)$ shows that

$$
(0) \longrightarrow \mathfrak{F}\left(\Omega_{G_{r} T}^{2}\left(\widehat{Z}_{r}(\lambda)\right)\right) \longrightarrow \mathfrak{F}(\widehat{E}) \longrightarrow Z_{r}(\lambda) \longrightarrow(0)
$$

is the almost split sequence terminating in $Z_{r}(\lambda)$. In view of Theorem 4.2, Lemma $3.2(3)$ and [2, (V.1.16)], its middle term $E \cong \mathfrak{F}(\widehat{E})$ is indecomposable.

Theorem 4.4. Let $\lambda \in X(T)$. The baby Verma module $Z_{r}(\lambda)$ is either projective or quasi-simple.

Proof. In view of Lemma 4.1 and Corollary 4.3 , it remains to rule out the case where $\Theta_{r}(\lambda) \cong$ $\mathbb{Z}\left[D_{\infty}\right]$.

Let $\widehat{\Theta}_{r}(\lambda) \subseteq \Gamma_{s}\left(G_{r} T\right)$ be the component containing the vertex $\left[\widehat{Z}_{r}(\lambda)\right]$. Thanks to Lemma $3.3(2)$, we have $\Theta_{r}(\lambda)=\mathfrak{F}\left(\widehat{\Theta}_{r}(\lambda)\right)$. Assuming $\Theta_{r}(\lambda) \cong \mathbb{Z}\left[D_{\infty}\right]$, we consider the almost split sequence

$$
(0) \longrightarrow \Omega_{G_{r} T}^{2}\left(\widehat{Z}_{r}(\lambda)\right) \longrightarrow \widehat{E} \longrightarrow \widehat{Z}_{r}(\lambda) \longrightarrow(0)
$$

terminating in $\widehat{Z}_{r}(\lambda)$. By assumption, there exists an indecomposable $G_{r}$-module $Y_{r}(\lambda) \nRightarrow Z_{r}(\lambda)$ such that $[E]:=[\mathfrak{F}(\widehat{E})]$ is the only predecessor of $\left[Y_{r}(\lambda)\right] \in \Theta_{r}(\lambda)$. Let $\left[\widehat{Y}_{r}(\lambda)\right] \in \widehat{\Theta}_{r}(\lambda)$ be a pre-image of $\left[Y_{r}(\lambda)\right]$ under $\mathfrak{F}$, and consider the almost split sequence

$$
(* *) \quad(0) \longrightarrow \Omega_{G_{r} T}^{2}\left(\widehat{Y}_{r}(\lambda)\right) \longrightarrow \widehat{D} \longrightarrow \widehat{Y}_{r}(\lambda) \longrightarrow(0)
$$

terminating in $\widehat{Y}_{r}(\lambda)$.

We now proceed in several steps:

(i) We have $\widehat{D} \cong \widehat{E}$.

Lemma $3.2(3)$ provides isomorphisms $\mathfrak{F}(\widehat{D}) \cong E \cong \mathfrak{F}(\widehat{E})$, and [26, (4.1)] implies the existence of $\mu \in X(T)$ such that $\widehat{D} \cong \widehat{E} \otimes_{k} k_{\mu}$.

Since $\mathfrak{F}$ sends the $\tau_{G_{r} T}$-orbits in $\widehat{\Theta}_{r}(\lambda)$ onto the $\tau_{G_{r}}$-orbits in $\Theta_{r}(\lambda)$ (see Lemma 3.2), we conclude that the component $\widehat{\Theta}_{r}(\lambda)$ has infinitely many $\tau_{G_{r} T}$-orbits. Owing to Proposition 3.4, the tree class $\bar{T}_{\widehat{\Theta}_{r}(\lambda)}$ is therefore isomorphic to $A_{\infty}, A_{\infty}^{\infty}$, or $D_{\infty}$. However, in components with the former two tree types, every vertex has at most two successors. Thanks to Lemma 3.3, the component $\Theta_{r}(\lambda)$ would also enjoy this property. As $\Theta_{r}(\lambda) \cong \mathbb{Z}\left[D_{\infty}\right]$, this is not possible, so that $\widehat{\Theta}_{r}(\lambda)$ also has tree class $D_{\infty}$.

By general theory, the auto-equivalence $[M] \mapsto\left[M \otimes_{k} k_{\mu}\right]$ induces an automorphism of $D_{\infty}$. As this map fixes the node corresponding to $\widehat{E}$, there exists $m_{\widehat{E}} \in \mathbb{Z}$ such that $\tau_{G_{r} T}^{m_{\widehat{E}}}(\widehat{E}) \cong \widehat{E} \otimes_{k} k_{\mu}$. Application of $\mathfrak{F}$ gives

$$
\Omega_{G_{r}}^{2 m_{\widehat{E}}}(E) \cong \mathfrak{F}\left(\widehat{E} \otimes_{k} k_{\mu}\right) \cong \mathfrak{F}(\widehat{D}) \cong E .
$$

The assumption $m_{\widehat{E}} \neq 0$ thus yields the periodicity of $E$, whence

$$
1=\operatorname{dim} V_{r}(G)_{E}=\operatorname{dim} V_{r}(G)_{\Theta_{r}(\lambda)}=\operatorname{dim} V_{r}(G)_{Z_{r}(\lambda)} .
$$

We may now apply Lemma 4.1 to reach a contradiction. Consequently, $m_{\widehat{E}}=0$, whence $\widehat{E} \cong$ $\widehat{E} \otimes_{k} k_{\mu} \cong \widehat{D}$.

(ii) We have $\left[\widehat{Y}_{r}(\lambda): \widehat{Z}_{r}(\lambda)\right]=1$.

Consider the almost split sequence

$$
(0) \longrightarrow \Omega_{G_{r} T}^{2}\left(\widehat{Y}_{r}(\lambda)\right) \longrightarrow \widehat{E} \longrightarrow \widehat{Y}_{r}(\lambda) \longrightarrow(0) .
$$


By virtue of Lemma 3.8 and Theorem $4.2(1)$, the assumption $\left(\widehat{Y}_{r}(\lambda): \widehat{Z}_{r}(\lambda)\right)=0$ implies

$$
\operatorname{supp}_{\Delta}\left(\widehat{Y}_{r}(\lambda)\right) \subseteq X(T)_{>\lambda}
$$

Thus, by Lemma [3.9, the highest weights $\mu$ of the constituents $\widehat{L}_{r}(\mu)$ of $\operatorname{Top}\left(\widehat{Y}_{r}(\lambda)\right)$ belong to $X(T)_{>\lambda}$. Owing to Lemma 3.1, the projective cover $\widehat{P}\left(\widehat{Y}_{r}(\lambda)\right)$ of $\widehat{Y}_{r}(\lambda)$ satisfies $\operatorname{supp}_{\Delta}\left(\widehat{P}\left(\widehat{Y}_{r}(\lambda)\right) \subseteq\right.$ $X(T)_{>\lambda}$ and, thanks to Lemma 3.8, the submodule $\Omega_{G_{r} T}\left(\widehat{Y}_{r}(\lambda)\right)$ inherits this property. A repetition of this argument implies $\operatorname{supp}_{\Delta}\left(\Omega_{G_{r} T}^{2}\left(\widehat{Y}_{r}(\lambda)\right)\right) \subseteq X(T)_{>\lambda}$. In virtue of Lemma 3.8, our almost split sequence then yields

$$
\left[\widehat{E}: \widehat{Z}_{r}(\lambda)\right]=\left[\Omega_{G_{r} T}^{2}\left(\widehat{Y}_{r}(\lambda)\right): \widehat{Z}_{r}(\lambda)\right]+\left[\widehat{Y}_{r}(\lambda): \widehat{Z}_{r}(\lambda)\right]=0
$$

which contradicts Theorem $4.2(1)$. Consequently, $\left[\widehat{Y}_{r}(\lambda): \widehat{Z}_{r}(\lambda)\right] \geq 1$, and the reverse inequality follows from Lemma 3.8 and Theorem $4.2(1)$.

(iii) There exists a surjection $g: \widehat{Y}_{r}(\lambda) \longrightarrow \widehat{Z}_{r}(\lambda)$.

In virtue of Theorem 4.2 and (ii), we have $\operatorname{supp}_{\Delta}\left(\widehat{Y}_{r}(\lambda)\right) \subseteq X(T)_{\geq \lambda}$ as well as $\left[\widehat{Y}_{r}(\lambda): \widehat{Z}_{r}(\lambda)\right]=1$. Consequently, there exist submodules $N \subseteq M \subseteq \widehat{Y}_{r}(\lambda)$ such that

(a) $M / N \cong \widehat{Z}_{r}(\lambda)$, and

(b) $\widehat{Y}_{r}(\lambda) / M$ belongs to $\mathcal{F}(\Delta)$ with $\operatorname{supp}_{\Delta}\left(\widehat{Y}_{r}(\lambda) / M\right) \subseteq X(T)_{>\lambda}$.

There results an exact sequence

$$
(0) \longrightarrow \widehat{Z}_{r}(\lambda) \longrightarrow \widehat{Y}_{r}(\lambda) / N \longrightarrow \widehat{Y}_{r}(\lambda) / M \longrightarrow(0) .
$$

Lemma 3.1 implies $\operatorname{Ext}_{G_{r} T}^{1}\left(\widehat{Z}_{r}(\mu), \widehat{Z}_{r}(\lambda)\right)=(0)$ for all $\mu \in X(T)_{>\lambda}$. Hence our sequence splits, and

$$
\widehat{Y}_{r}(\lambda) / N \cong\left(\widehat{Y}_{r}(\lambda) / M\right) \oplus \widehat{Z}_{r}(\lambda) \text {. }
$$

We may now define $g:=\operatorname{pr} \circ \pi$ to be the composite of the projection pr $: \widehat{Y}_{r}(\lambda) / N \longrightarrow \widehat{Z}_{r}(\lambda)$ with the canonical map $\pi: \widehat{Y}_{r}(\lambda) \longrightarrow \widehat{Y}_{r}(\lambda) / N$.

(iv) We have $\operatorname{dim}_{k} \operatorname{Hom}_{G_{r} T}\left(\widehat{E}, \widehat{Z}_{r}(\lambda)\right)=1$.

We write $X:=\Omega_{G_{r} T}^{2}\left(\widehat{Z}_{r}(\lambda)\right)$ for notational convenience. Application of the left exact functor $\operatorname{Hom}_{G_{r} T}\left(-, \widehat{Z}_{r}(\lambda)\right)$ to the almost split sequence $(*)$ terminating in $\widehat{Z}_{r}(\lambda)$ gives an exact sequence

$$
(0) \longrightarrow \operatorname{Hom}_{G_{r} T}\left(\widehat{Z}_{r}(\lambda), \widehat{Z}_{r}(\lambda)\right) \longrightarrow \operatorname{Hom}_{G_{r} T}\left(\widehat{E}, \widehat{Z}_{r}(\lambda)\right) \longrightarrow \operatorname{Hom}_{G_{r} T}\left(X, \widehat{Z}_{r}(\lambda)\right) \text {. }
$$

Since $\operatorname{Top}_{G_{r} T}(X)$ is a direct summand of $\bigoplus_{\mu>\lambda}\left[X: \widehat{Z}_{r}(\mu)\right] \widehat{L}_{r}(\mu)$, and all weights of $\widehat{Z}_{r}(\lambda)$ belong to $X(T)_{\leq \lambda}$, it follows that any homomorphism $X \longrightarrow \widehat{Z}_{r}(\lambda)$ sends the generators of $X$ to zero. Consequently, $\operatorname{Hom}_{G_{r} T}\left(X, \widehat{Z}_{r}(\lambda)\right)=(0)$, implying

$$
\operatorname{Hom}_{G_{r} T}\left(\widehat{E}, \widehat{Z}_{r}(\lambda)\right) \cong \operatorname{Hom}_{G_{r} T}\left(\widehat{Z}_{r}(\lambda), \widehat{Z}_{r}(\lambda)\right) \cong k
$$

as desired.

Let $f: \widehat{E} \longrightarrow \widehat{Z}_{r}(\lambda)$ and $h: \widehat{E} \longrightarrow \widehat{Y}_{r}(\lambda)$ be the irreducible morphisms given by the almost split sequences $(*)$ and $(* *)$, terminating in $\widehat{Z}_{r}(\lambda)$ and $\widehat{Y}_{r}(\lambda)$, respectively. By virtue of (iii) and (iv), there exists $\alpha \in k \backslash\{0\}$ such that

$$
g \circ h=\alpha f .
$$

Since $\alpha f$ is irreducible and $h$ is not split injective, the map $g$ is split surjective, 2, (V.5)]. Consequently, $\widehat{Z}_{r}(\lambda) \cong \widehat{Y}_{r}(\lambda)$, a contradiction.

Corollary 4.5. Given $\lambda \in X(T)$, the module $\widehat{Z}_{r}(\lambda)$ is either projective or quasi-simple. 
Proof. Assume that $\widehat{Z}_{r}(\lambda)$ is not projective. Thanks to Theorem 4.2, it suffices to show that the tree class $\widehat{T}_{r}(\lambda)$ of the component $\widehat{\Theta}_{r}(\lambda) \subseteq \Gamma_{s}\left(G_{r} T\right)$ containing $\left[\widehat{Z}_{r}(\lambda)\right]$ is isomorphic to $A_{\infty}$.

By Theorem 4.4. the component $\Theta_{r}(\lambda)$ has tree class $A_{\infty}$ and thus has infinitely many $\tau_{G_{r}-}$ orbits. In view of Lemma 3.3. the component $\widehat{\Theta}_{r}(\lambda)$ also has infinitely many $\tau_{G_{r} T^{-o r b i t s} \text {, and an }}$ application of Proposition 3.4 now implies $\widehat{T}_{r}(\lambda)=A_{\infty}, A_{\infty}^{\infty}$, or $D_{\infty}$. By Theorem 4.2 and the proof of Theorem 4.4, the latter two alternatives cannot occur.

Remark. Aside from simple modules and baby Verma modules, the so-called Weyl modules $V(\lambda)$ (see [35, (II.2.13)]) and their duals form another important class of $G_{r}$-modules. For the group scheme $\operatorname{SL}(2)_{1}$, these modules belong to components of type $\mathbb{Z}\left[\tilde{A}_{12}\right]$, cf. [20, (4.3.1)]. Since the support varieties of Weyl modules are $G$-invariant, they will in general have dimension $\geq 3$, so that [18, (2.2)] ensures that Weyl modules usually belong to components of type $\mathbb{Z}\left[A_{\infty}\right]$. However, their position within these components is not known.

\section{VARIETIES fOr Verma Modules}

We begin by generalizing [22, (2.3)] to Verma modules of higher Frobenius kernels. Recall that $h$ is the Coxeter number of the reductive group $G$. Unless mentioned otherwise, the prime $p$ is assumed to be good for $G$.

The interested reader may compare the following result with [41, (4.1.2)]. Under the additional hypothesis of $\mathcal{V}_{U_{r}}(k)$ being irreducible, the implication $(3) \Rightarrow(1)$ provides the converse to part (b) of that result. Also note that the assumption $p \geq h$ implies that $\mathcal{V}_{U_{1}}(k)$ is irreducible.

Lemma 5.1. Suppose $p \geq h$ and that $\mathcal{V}_{U_{r}}(k)$ is irreducible. Let $r \leq s$ and $\lambda \in X(T)$ be a weight. Then the following statements are equivalent:

(1) $\mathcal{V}_{G_{r}}\left(Z_{s}(\lambda)\right)=\mathcal{V}_{U_{r}}(k)$.

(2) $\operatorname{cx}_{G_{r}}\left(Z_{s}(\lambda)\right)=\operatorname{dim} \mathcal{V}_{U_{r}}(k)$.

(3) $\lambda$ is p-regular.

Proof. (1) $\Rightarrow(2)$. Thanks to [25, (1.5.2)] the cohomology ring $\mathrm{H}^{\mathrm{ev}}\left(G_{r}, k\right)$ is finitely generated. Thus, the arguments of [8, (2.3)] show that the complexity of the $G_{r}$-module $Z_{s}(\lambda)$ coincides with the dimension of $\mathcal{V}_{G_{r}}\left(Z_{s}(\lambda)\right)$.

$(2) \Rightarrow(3)$. The $G_{r} T$-module $\widehat{Z}_{s}(\lambda)$ is $B_{r}^{-}$-projective, and [35, (II.11.2)] provides a $\widehat{Z}_{r}$-filtration of $\left.\widehat{Z}_{s}(\lambda)\right|_{G_{r} T}$. Applying the exact functor $\mathfrak{F}$, we conclude that $\left.Z_{s}(\lambda)\right|_{G_{r}}$ has a $Z_{r}$-filtration, so that Proposition 1.2 gives

$$
\mathcal{V}_{G_{r}}\left(Z_{s}(\lambda)\right) \subseteq \mathcal{V}_{U_{r}}(k)
$$

for any $\lambda \in X(T)$. Since the variety $\mathcal{V}_{U_{r}}(k)$ is irreducible, $(2)$ implies that the above inclusion is in fact an equality. In view of [41, (4.1.2)], we obtain the $p$-regularity of $\lambda$.

$(3) \Rightarrow(1)$. Following the proof of [22, (2.3)], we first consider the case where $\lambda=0$. The augmentation map $\varepsilon: \operatorname{Dist}\left(G_{r}\right) \longrightarrow k$ furnishes a split surjective homomorphism

$$
f: Z_{s}(0) \longrightarrow k ; u \otimes \alpha \mapsto \varepsilon(u) \alpha
$$

of $\operatorname{Dist}\left(U_{r}\right)$-modules. Thus, $k$ is a direct summand of $\left.Z_{s}(0)\right|_{U_{r}}$, so that $\mathcal{V}_{U_{r}}(k) \subseteq \mathcal{V}_{U_{r}}\left(Z_{s}(0)\right)$. The foregoing observations now yield

$$
\mathcal{V}_{G_{r}}\left(Z_{s}(0)\right)=\mathcal{V}_{U_{r}}(k)
$$


To transfer this result to other $p$-regular weights, we shall use translation functors $T_{\lambda}^{\mu}$, cf. 35 , (II.9.22)]. Their definition in conjuction with [51, (7.2)], yields

$$
\mathcal{V}_{G_{r}}\left(T_{\lambda}^{\mu}(M)\right) \subseteq \mathcal{V}_{G_{r}}(M)
$$

Now let $\lambda$ be another weight in the standard alcove

$$
C_{0}:=\left\{\lambda \in X(T) \otimes_{\mathbb{Z}} \mathbb{R} \mid 0<\left\langle\lambda+\rho, \alpha^{\vee}\right\rangle<p \quad \forall \alpha \in \Psi^{+}\right\}
$$

(such weights are necessarily $p$-regular). The translation functor $T_{0}^{\lambda}$ provides a categorical equivalence between the blocks of $\operatorname{Dist}\left(G_{r}\right)$ associated to $\lambda$ and 0 and sends $Z_{s}(0)$ onto $Z_{s}(\lambda)$ (cf. 35. (II.9.22(1)-(3))]). Consequently, the $G_{r^{-}}$-support varieties of $Z_{s}(\lambda)$ and $Z_{s}(0)$ are equal.

By the same token, $\mathcal{V}_{G_{r}}\left(Z_{s}(\lambda)\right)$ and $\mathcal{V}_{G_{r}}\left(Z_{s}(\gamma)\right)$ coincide, whenever $\lambda$ and $\gamma$ are $p$-regular elements belonging to the same alcove.

Let $C$ be an alcove such that $\mathcal{V}_{G_{r}}\left(Z_{s}(\lambda)\right)=\mathcal{V}_{U_{r}}(k)$ for some (and hence every) $p$-regular element $\lambda \in C$. We choose an alcove $D$ adjacent to $C$ and let $s_{F}$ be the unique affine reflection relative to the adjoining wall $\bar{F}=\bar{C} \cap \bar{D}$ (facet of codimension 1). Note that $s_{F} \cdot \lambda \in D$ is a $p$-regular element. Next, we choose an element $w \in W_{p}$ such that $w \cdot C=C_{0}$. Then $w \cdot D$ is adjacent to $C_{0}$ with adjoining wall $w \cdot F$ and associated reflection $s^{\prime}=w s_{F} w^{-1}$. Observe that $w^{-1} \cdot s^{\prime} \cdot(w \cdot \lambda)=s_{F} \cdot \lambda$. Now let $\mu \in w \cdot F$ be a weight on the wall $w \cdot F$. Suppose that $\lambda<s_{F} \cdot \lambda$. An application of 35, (II.9.22(3))] provides an exact sequence

$$
(0) \longrightarrow Z_{s}(\lambda) \longrightarrow T_{\mu}^{w \cdot \lambda}\left(Z_{s}\left(w^{-1} \cdot \mu\right)\right) \longrightarrow Z_{s}\left(s_{F} \cdot \lambda\right) \longrightarrow(0)
$$

of $G_{s}$-modules, so that

$$
\mathcal{V}_{G_{r}}\left(Z_{s}(\lambda)\right) \subseteq \mathcal{V}_{G_{r}}\left(T_{\mu}^{w \cdot \lambda}\left(Z_{s}\left(w^{-1} \cdot \mu\right)\right)\right) \cup \mathcal{V}_{G_{r}}\left(Z_{s}\left(s_{F} \cdot \lambda\right)\right) \subseteq \mathcal{V}_{G_{r}}\left(Z_{s}\left(w^{-1} \cdot \mu\right)\right) \cup \mathcal{V}_{G_{r}}\left(Z_{s}\left(s_{F} \cdot \lambda\right)\right) .
$$

Since $\mathcal{V}_{G_{r}}\left(Z_{s}(\lambda)\right)=\mathcal{V}_{U_{r}}(k)$ is irreducible and $w^{-1} \cdot \mu \in w \cdot F$ is not $p$-regular, we have $\mathcal{V}_{G_{r}}\left(Z_{s}(\lambda)\right)=$ $\mathcal{V}_{G_{r}}\left(Z_{s}\left(s_{F} \cdot \lambda\right)\right)$, cf. [41, (4.1.2)]. Therefore, $\mathcal{V}_{G_{r}}\left(Z_{s}(\gamma)\right)=\mathcal{V}_{U_{r}}(k)$ for any $p$-regular element $\gamma \in D$. The case $\lambda>s_{F} \cdot \lambda$ can be treated similarly.

Since $W_{p}$ acts transitively on the set of alcoves, we are done.

We shall use the foregoing result mainly for Levi subgroups defined by simple roots. The following example provides another case, where $\mathcal{V}_{U_{r}}(k)$ is irreducible.

Example. Suppose that $p \geq 3$ and consider the group $G=\operatorname{SL}(3)$. We let $U$ denote the unipotent subgroup of upper triangular matrices. Then $\mathfrak{u}:=\operatorname{Lie}(U)$ is isomorphic to the 3-dimenisonal $p$ unipotent Heisenberg algebra with trivial $p$-map and multiplication relative to the standard basis $\{a, b, c\}$ defined via

$$
[a, b]=c \text { and }[a, c]=0=[b, c] .
$$

For $r \geq 2$ and any subspace $\mathfrak{v} \subseteq \mathfrak{u}$, we consider the commuting variety

$$
\mathcal{C}^{r}(\mathfrak{v}):=\left\{\left(v_{0}, \ldots, v_{r-1}\right) \in \mathfrak{v}^{r} \mid\left[v_{i}, v_{j}\right]=0 \text { for } i \neq j\right\} .
$$

Writing $v_{j}:=x_{j} a+y_{j} b+z_{j} c$, we obtain

$$
(*) \quad\left[v_{i}, v_{j}\right]=\left(x_{i} y_{j}-x_{j} y_{i}\right) c
$$

Thus, for $\mathfrak{v}:=k a \oplus k b$, the variety $\mathcal{C}^{r}(\mathfrak{v})$ is defined by the determinantal ideal

$$
I_{2}(A) \subseteq k\left[X_{0}, \ldots, X_{r-1}, Y_{0}, \ldots, Y_{r-1}\right],
$$

given by the $(2 \times 2)$-minors of the $(r \times r)$-matrix

$$
A:=\left(\begin{array}{ccc}
X_{0} & \ldots & X_{r-1} \\
Y_{0} & \ldots & Y_{r-1}
\end{array}\right)
$$


Thanks to [7, (7.3.1)], the determinantal variety $C^{r}(\mathfrak{v})$ is irreducible.

In view of $(*)$, the isomorphism

$$
\mathfrak{v}^{r} \times(k c)^{r} \longrightarrow \mathfrak{u}^{r} ;\left(v_{0}, \ldots, v_{r-1}, w_{0}, \ldots, w_{r-1}\right) \mapsto\left(v_{0}+w_{0}, \ldots, v_{r-1}+w_{r-1}\right)
$$

maps the irreducible variety $\mathcal{C}^{r}(\mathfrak{v}) \times(k c)^{r}$ onto $\mathcal{C}^{r}(\mathfrak{u})$, so that $\mathcal{C}^{r}(\mathfrak{u})$ is also irreducible. By applying [50, (1.7),(1.8)] and [51, (5.2)] successively, we conclude that the support variety $\mathcal{V}_{U_{r}}(k)$ is irreducible. Owing to [7, (7.3.1)], we also have $\operatorname{dim} \mathcal{V}_{U_{r}}(k)=2 r+1$.

Given a root $\alpha \in \Psi$, we let $U_{\alpha} \subseteq G$ denote the corresponding root subgroup, see [35, (II.1.2)].

Corollary 5.2. Let $\lambda \in X(T)$ be a weight such that $\Psi_{\lambda} \neq \Psi$. Given $r \leq s$, we have $\Sigma \cap\left(\Psi \backslash \Psi_{\lambda}\right) \neq \emptyset$ as well as

$$
\mathcal{V}_{\left(U_{\alpha}\right)_{r}}(k) \subseteq \mathcal{V}_{G_{r}}\left(Z_{s}(\lambda)\right) \quad \forall \alpha \in \Sigma \cap\left(\Psi \backslash \Psi_{\lambda}\right)
$$

In particular, $\operatorname{cx}_{G_{r}}\left(Z_{s}(\lambda)\right) \geq r$.

Proof. Since $\Psi_{\lambda} \subseteq \Psi$ is a subsystem ([33, (2.7)]), there exists a simple root $\alpha$ in $\Psi \backslash \Psi_{\lambda}$. Given such a root $\alpha$, we let $L \supseteq T$ be the Levi subgroup of $G$ defined by $\alpha$. Thanks to Proposition 1.2 , the variety $\mathcal{V}_{G_{s}}\left(Z_{s}(\lambda)\right)$ contains $\mathcal{V}_{L_{s}}\left(Z_{s}^{L}(\lambda)\right)$, so that

$$
\mathcal{V}_{L_{r}}\left(Z_{s}^{L}(\lambda)\right) \subseteq \mathcal{V}_{G_{r}}\left(Z_{s}(\lambda)\right)
$$

By assumption, the weight $\lambda$ is $p$-regular on $L$. Observe that the root group $U_{\alpha}$ is the unipotent radical of the Borel subgroup $B \cap L$ of $L$. Moreover, $U_{\alpha}$ is isomorphic to the additive group $\mathbb{G}_{a} \cong \operatorname{Spec}_{k}(k[T])$. Accordingly, we have $\left(U_{\alpha}\right)_{r} \cong \mathbb{G}_{a(r)}$, whence

$$
\mathcal{V}_{\left(U_{\alpha}\right)_{r}}(k) \cong \mathcal{V}_{\mathbb{G}_{a(r)}}(k) \cong k^{r}
$$

is irreducible, see [11, (4.1)]. Consequently, Lemma 5.1 yields $\mathcal{V}_{L_{r}}\left(Z_{s}^{L}(\lambda)\right)=\mathcal{V}_{\left(U_{\alpha}\right)_{r}}(k)$, whence $\mathcal{V}_{\left(U_{\alpha}\right)_{r}}(k) \subseteq \mathcal{V}_{G_{r}}\left(Z_{s}(\lambda)\right)$ and $\operatorname{cx}_{G_{r}}\left(Z_{s}(\lambda)\right) \geq \operatorname{dim} \mathcal{V}_{\left(U_{\alpha}\right)_{r}}(k)=r$

Corollary 5.3. Suppose that $p \geq 5$. Let $\lambda \in X(T)$ be a weight such that

(a) $\Psi_{\lambda} \neq \Psi$, and

(b) $\operatorname{cx}_{G_{r}}\left(Z_{r}(\lambda)\right)=r$.

Then there exists a simple root $\alpha \in \Sigma$ such that $\mathcal{V}_{G_{r}}\left(Z_{r}(\lambda)\right)=\mathcal{V}_{\left(U_{\alpha}\right)_{r}}(k)$ is irreducible.

Proof. According to Corollary [5.2, we can find a simple root $\alpha \in \Psi$ such that the $r$-dimensional irreducible variety $\mathcal{V}_{\left(U_{\alpha}\right)_{r}}(k)$ is contained in $\mathcal{V}_{G_{r}}\left(Z_{r}(\lambda)\right)$. By the arguments of Lemma 4.1, this implies

$$
V_{r}\left(U_{\alpha}\right) \subseteq V_{r}(G)_{Z_{r}(\lambda)}
$$

In view of (b), the rank variety $V_{r}\left(U_{\alpha}\right)$ is an irreducible component of the $B$-invariant variety $V_{r}(G)_{Z_{r}(\lambda)}$. Thus, the stabilizer $H \subseteq B$ of $V_{r}\left(U_{\alpha}\right)$ is a closed subgroup of finite index, and the connectedness of $B$ gives $H=B$, so that the variety $V_{r}\left(U_{\alpha}\right)$ is also $B$-invariant. In particular, the $B$-orbit of the canonical isomorphism $\mathbb{G}_{a(r)} \stackrel{\sim}{\longrightarrow}\left(U_{\alpha}\right)_{r}$ is contained in $V_{r}\left(U_{\alpha}\right)$, implying the $B$-invariance of $\left(U_{\alpha}\right)_{r} \subseteq B_{r}$. Thus, $\mathfrak{g}_{\alpha}=\operatorname{Lie}\left(\left(U_{\alpha}\right)_{r}\right)$ enjoys the same property, and the proof of 22 , (3.3)] provides a decomposition $G=S H$ of $G$ as an almost direct product with

(1) $\mathfrak{g}_{\alpha} \subseteq \operatorname{Lie}(S)$, and

(2) $S$ is almost simple of type $A_{1}$, and

(3) $\mathfrak{g}=\operatorname{Lie}(S) \oplus \operatorname{Lie}(H)$. 
In view of (3), we have $(S \cap H)_{1} \subseteq \operatorname{Cent}(S)_{1}=e_{k}$, so that $(S \cap H)_{r}=e_{k}$. Thus, the canonical map $S \times H \longrightarrow G$ induces a closed embedding $S_{r} \times H_{r} \hookrightarrow G_{r}$. Applying [35, (I.9.6)] several times, we obtain

$$
\operatorname{dim}_{k} k\left[G_{r}\right]=p^{r \operatorname{dim} G}=p^{r(\operatorname{dim} S+\operatorname{dim} H)}=\operatorname{dim}_{k} k\left[S_{r}\right] \operatorname{dim}_{k} k\left[H_{r}\right]=\operatorname{dim}_{k} k\left[S_{r} \times H_{r}\right],
$$

so that the above map is in fact an isomorphism.

In view of [35, (I.7.9(3))], the arguments of [22, (1.1)] show that the decomposition $G_{r}=S_{r} \times H_{r}$ induces an isomorphism

$$
Z_{r}(\lambda) \cong Z_{r}^{S}\left(\lambda^{\prime}\right) \otimes_{k} Z_{r}^{H}\left(\lambda^{\prime \prime}\right)
$$

between $Z_{r}(\lambda)$ and the outer tensor product of the corresponding Verma modules of the factors. The formula for varieties of outer tensor products, established in the proof of [51, (7.2)], implies

$$
(*) \quad \mathcal{V}_{G_{r}}\left(Z_{r}(\lambda)\right) \cong \mathcal{V}_{S_{r}}\left(Z_{r}^{S}\left(\lambda^{\prime}\right)\right) \times \mathcal{V}_{H_{r}}\left(Z_{r}^{H}\left(\lambda^{\prime \prime}\right)\right) .
$$

Since $\mathfrak{g}_{\alpha} \subseteq \operatorname{Lie}(S)$, we see that $\alpha$ is a root of $S$. Consequently, $U_{\alpha} \subseteq S$, and Corollary 5.2 gives

$$
\mathcal{V}_{\left(U_{\alpha}\right)_{r}}(k) \subseteq \mathcal{V}_{S_{r}}\left(Z_{r}^{S}\left(\lambda^{\prime}\right)\right) \subseteq \mathcal{V}_{\left(U_{\alpha}\right)_{r}}(k)
$$

Consequently, $\operatorname{dim} \mathcal{V}_{S_{r}}\left(Z_{r}^{H}(\lambda)\right)=0$, so that the conical variety $\mathcal{V}_{S_{r}}\left(Z_{r}^{H}(\lambda)\right)$ is a singleton. The isomorphism $(*)$ now implies that the variety $\mathcal{V}_{G_{r}}\left(Z_{r}(\lambda)\right)$ is irreducible. Hence the inclusion $\mathcal{V}_{\left(U_{\alpha}\right)_{r}}(k) \subseteq \mathcal{V}_{G_{r}}\left(Z_{r}(\lambda)\right)$ is in fact an equality.

Remarks. (1) The case $r=1$ is of course a direct consequence of Carlson's Theorem [9, Thm. 1], which also holds in our context (cf. [51, (7.7)]).

(2) If $G$ is almost simple, then the proof of (5.3) yields that $G$ is of type $A_{1}$. In that case, Corollary 5.2 and Proposition 1.2 show that $Z_{r}(\lambda)$ has an irreducible support variety of dimension $r$, whenever $\Psi_{\lambda} \neq \Psi$.

(3) The reader is referred to the example at the end of Section 6 for a discussion of periodic baby Verma modules.

Given $\lambda \in X(T)$ with $\left\langle\lambda+\rho, \alpha^{\vee}\right\rangle \neq 0$ for some $\alpha \in \Psi$, we define the depth of $\lambda$ via

$$
\operatorname{dp}(\lambda):=\min \left\{s \in \mathbb{N}_{0} \mid \Psi_{\lambda}^{s} \neq \Psi\right\},
$$

and put $\operatorname{dp}(\lambda)=-\infty$ otherwise. Let $\lambda \in X(T)$ and denote by $\mathcal{B}_{r}(\lambda)$ the set of weights of the simple $\operatorname{Dist}\left(G_{r}\right)$-modules belonging to the block containing the simple $\operatorname{Dist}\left(G_{r}\right)$-module $L_{r}(\lambda)$. Thanks to [32, (5.5)] (see also [35, (II.9.14), (II.9.22)]), we have

$$
\mathcal{B}_{r}(\lambda)=W \cdot \lambda+p^{\operatorname{dp}(\lambda)} \mathbb{Z} \Psi+p^{r} X(T) .
$$

(Here we employ the convention $p^{-\infty}=0$.) The notion of the depth of a weight will also play an important role in Section 6 below.

Proposition 5.4. Suppose that $G$ is defined over $\mathbb{F}_{p}$. Let $r \leq s$ and set $t=(s-r) \operatorname{dim} \mathfrak{u}$. For every weight $\lambda=\lambda_{0}+p^{r} \lambda_{1} \in X(T)$ with $\lambda_{0} \in X_{r}(T)$, the $G_{r} T$-module $\left.\widehat{Z}_{s}(\lambda)\right|_{G_{r} T}$ possesses a filtration

$$
(0)=M_{0} \subseteq M_{1} \subseteq \cdots \subseteq M_{p^{t}}=\left.\widehat{Z}_{s}(\lambda)\right|_{G_{r} T}
$$

by $G_{r} T$-submodules, such that $M_{i} / M_{i-1} \cong \widehat{Z}_{r}\left(\gamma_{i}\right)$ for some $\gamma_{i} \in \mathcal{B}_{r}\left(\lambda_{0}\right)$. 
Proof. We first assume $(G, G)$ to be simply connected, so that every weight $\gamma \in X(T)$ is of the form

$$
\gamma=\gamma_{0}+p^{r} \gamma_{1}
$$

with $\gamma_{0} \in X_{r}(T)$ and $\gamma_{1} \in X(T)$ (cf. [35, (II.9.14)]).

Since $\widehat{Z}_{s}(\lambda)$ is $B_{r}^{-}$-projective, [35, (II.11.2)] provides a $\widehat{Z}_{r}$-filtration of $\left.\widehat{Z}_{s}(\lambda)\right|_{G_{r} T}$ of length $p^{t}$. Let $\gamma \in X(T)$ be a weight, written as

$$
\gamma=\mu+p^{s} \nu
$$

with $\mu \in X_{s}(T)$. In view of [35, (II.9.6(6))], we have

$$
\widehat{L}_{s}(\gamma) \cong \widehat{L}_{s}(\mu) \otimes_{k} k_{p^{s} \nu}
$$

We now decompose $\mu \in X_{s}(T)$ as $\mu=\gamma_{0}+p^{r} \gamma_{1}$ with $\gamma_{0} \in X_{r}(T)$ and $\gamma_{1} \in X(T)$. The assumption $\left\langle\gamma_{1}, \alpha^{\vee}\right\rangle<0$ for some $\alpha \in \Sigma$ implies $\left\langle\mu, \alpha^{\vee}\right\rangle=\left\langle\gamma_{0}, \alpha^{\vee}\right\rangle+p^{r}\left\langle\gamma_{1}, \alpha^{\vee}\right\rangle\left\langle\left\langle\gamma_{0}, \alpha^{\vee}\right\rangle-p^{r}<0\right.$, a contradiction. Consequently, $\gamma_{1} \in X(T)_{+}$, and Steinberg's tensor product theorem [35, (II.3.16)] in conjunction with [35, (II.9.6g)] yields

$$
\left.\left.\left.\widehat{L}_{s}(\mu) \cong L(\mu)\right|_{G_{s} T} \cong L\left(\gamma_{0}\right)\right|_{G_{s} T} \otimes_{k} L\left(\gamma_{1}\right)^{[r]}\right|_{G_{s} T} .
$$

Upon restriction to $G_{r} T$, we obtain, observing [35, (II.9.6(6))],

$$
\begin{aligned}
\left.\widehat{L}_{s}(\gamma)\right|_{G_{r} T} & \left.\left.\left.\left.\left.\cong \widehat{L}_{s}(\mu)\right|_{G_{r} T} \otimes_{k} k_{p^{s}}\right|_{G_{r} T} \cong L\left(\gamma_{0}\right)\right|_{G_{r} T} \otimes_{k} L\left(\gamma_{1}\right)^{[r]}\right|_{G_{r} T} \otimes_{k} k_{p^{s} \nu}\right|_{G_{r} T} \\
& \cong \bigoplus_{\zeta \in X(T)} n_{\zeta} \widehat{L}_{r}\left(\gamma_{0}\right) \otimes_{k} k_{p^{r} \zeta+p^{s} \nu} \cong \bigoplus_{\zeta \in X(T)} n_{\zeta} \widehat{L}_{r}\left(\gamma_{0}+p^{r}\left(\zeta+p^{s-r} \nu\right)\right) \\
& \cong \bigoplus_{\zeta \in X(T)} m_{\zeta} \widehat{L}_{r}\left(\gamma_{0}+p^{r} \zeta\right),
\end{aligned}
$$

for some $n_{\zeta}, m_{\zeta} \in \mathbb{N}_{0}$.

Now let $\eta \in X_{r}(T)$ be an arbitrary weight. Thanks to [35, (II.11.2)], the factor $\widehat{Z}_{r}(\eta)$ occurs in the $\widehat{Z}_{r}$-filtration of $\left.\widehat{Z}_{s}(\lambda)\right|_{G_{r} T}$ with multiplicity

$$
\ell_{\eta}:=\operatorname{dim}_{k} \operatorname{Hom}_{G_{r} T}\left(\left.\widehat{Z}_{s}(\lambda)\right|_{G_{r} T}, \widehat{Z}_{r}^{\prime}(\eta)\right) .
$$

If $\widehat{L}_{s}(\gamma)$ is a composition factor of the $G_{s} T$-module $\widehat{Z}_{s}(\lambda)$, then $L_{s}(\gamma)$ is a composition factor of the indecomposable $G_{s}$-module $Z_{s}(\lambda)$, so that $\gamma$ belongs to $\mathcal{B}_{s}(\lambda)$. In view of the above isomorphisms, left exactness of $\operatorname{Hom}_{G_{r} T}\left(-, \widehat{Z}_{r}^{\prime}(\eta)\right)$ thus yields the estimate

$$
\ell_{\eta} \leq \sum_{\gamma \in \mathcal{B}_{s}(\lambda)} \sum_{\zeta \in X(T)} m_{\zeta, \gamma} \operatorname{dim}_{k} \operatorname{Hom}_{G_{r} T}\left(\widehat{L}_{r}\left(\gamma_{0}+p^{r} \zeta\right), \widehat{Z}_{r}^{\prime}(\eta)\right)
$$

According to [35, (II.9.6(2))], $\ell_{\eta} \neq 0$ implies that $\eta=\gamma_{0}+p^{r} \zeta$ for some $\gamma \in \mathcal{B}_{s}(\lambda), \zeta \in X(T)$.

By Jantzen's description $(\dagger)$ of $\mathcal{B}_{s}(\lambda)$ above, there exist $w \in W, \alpha_{0} \in \mathbb{Z} \Psi$, and $\chi \in X(T)$ such that

$$
\gamma=w \cdot \lambda+p^{\operatorname{dp}(\lambda)} \alpha_{0}+p^{s} \chi=w \cdot \lambda_{0}+p^{r} w\left(\lambda_{1}\right)+p^{\operatorname{dp}(\lambda)} \alpha_{0}+p^{s} \chi .
$$

Since $\lambda_{0} \in X_{r}(T)$, we have $\operatorname{dp}\left(\lambda_{0}\right) \leq r+1$. Consequently,

$$
\left\langle\lambda+\rho, \beta^{\vee}\right\rangle=\left\langle\lambda_{0}+\rho, \beta^{\vee}\right\rangle+p^{r}\left\langle\lambda_{1}, \beta^{\vee}\right\rangle \in p^{m} \mathbb{Z}+p^{r} \mathbb{Z} \subseteq p^{m} \mathbb{Z}
$$

for all $m \leq \operatorname{dp}\left(\lambda_{0}\right)-1$ and $\beta \in \Psi$. Thus, we have $\operatorname{dp}\left(\lambda_{0}\right) \leq \operatorname{dp}(\lambda)$ or $\operatorname{dp}(\lambda)=-\infty$.

Accordingly, setting $\beta_{0}:=p^{\operatorname{dp}(\lambda)-\operatorname{dp}\left(\lambda_{0}\right)} \alpha_{0}$ (with $\beta_{0}:=0$ for $\operatorname{dp}(\lambda)=-\infty$ ), the above identity attains the form

$$
\gamma=w \cdot \lambda_{0}+p^{\mathrm{dp}\left(\lambda_{0}\right)} \beta_{0}+p^{r} \omega
$$

for some $\omega \in X(T)$. This, however, implies that $\gamma=w \cdot \lambda_{0}+p^{\operatorname{dp}\left(\lambda_{0}\right)} \beta_{0}+p^{r} \omega$ belongs to $\mathcal{B}_{r}\left(\lambda_{0}\right)$. As a result, $\eta=\gamma_{0}+p^{r} \zeta=\gamma+p^{r}\left(\zeta-\gamma_{1}\right)$ enjoys the same property. 
In general, there exists a covering $\tilde{G}$ of $G$ such that the derived group $(\tilde{G}, \tilde{G})$ is simply connected. Our result now follows from Lemma 3.5 .

Corollary 5.5. Suppose that $G$ is defined over $\mathbb{F}_{p}$. Let $r \leq s$ and $\lambda=\lambda_{0}+p^{r} \lambda_{1}$ be a weight with $\lambda_{0} \in$ $X_{r}(T)$. The module $\left.Z_{s}(\lambda)\right|_{G_{r}}$ belongs to the block $\mathcal{B}_{r}\left(\lambda_{0}\right)$, and $\mathcal{V}_{G_{r}}\left(Z_{s}(\lambda)\right) \subseteq \bigcup_{\gamma \in \mathcal{B}_{r}\left(\lambda_{0}\right)} \mathcal{V}_{G_{r}}\left(Z_{r}(\gamma)\right)$.

Proof. This follows from Proposition 5.4 and standard properties of support varieties, see [41, (2.2.7)].

In the following, we let

$$
\operatorname{St}_{r}:=\operatorname{Dist}\left(G_{r}\right) \otimes_{\operatorname{Dist}\left(B_{r}\right)} k_{\left(p^{r}-1\right) \rho}
$$

denote the Steinberg module of $G_{r}$. Our next result generalizes [35, (II.11.8)].

Corollary 5.6. Suppose that $G$ is defined over $\mathbb{F}_{p}$. Let $r \leq s$. Then the following statements are equivalent:

(1) $\Psi_{\lambda}^{r}=\Psi$.

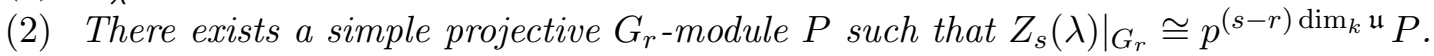

(3) $\left.Z_{s}(\lambda)\right|_{G_{r}}$ is projective.

Proof. In view of Lemma 3.5, we may assume that $(G, G)$ is simply connected.

$(1) \Rightarrow(2)$. Suppose that $\Psi_{\lambda}^{r}=\Psi$. Writing $\lambda=\lambda_{0}+p^{r} \lambda_{1}$, with $\lambda_{0} \in X_{r}(T)$, we obtain $\left\langle\lambda_{0}+\rho, \alpha^{\vee}\right\rangle=p^{r}$ for all $\alpha \in \Sigma$. Thanks to [35, (II.11.8)], this implies that $Z_{r}\left(\lambda_{0}\right)$ is a simple projective module and $\mathcal{B}_{r}\left(\lambda_{0}\right)=\lambda_{0}+p^{r} X(T)$. Thus, Proposition 5.4 in conjunction with [35, (II.3.7.(9))] provides a filtration of $\left.Z_{s}(\lambda)\right|_{G_{r}}$ with $Z_{r}\left(\lambda_{0}\right)$ being the only filtration factor. Consequently, $\left.Z_{s}(\lambda)\right|_{G_{r}} \cong p^{(s-r) \operatorname{dim}_{k} \mathfrak{u}} Z_{r}\left(\lambda_{0}\right)$.

$(2) \Rightarrow(3)$. This is trivial.

$(3) \Rightarrow(1)$. Let $\alpha \in \Sigma$ be a simple root of $\Psi$ and let $L$ be the corresponding Levi subgroup of $G$. By Proposition 1.2 we have

$$
\mathcal{V}_{L_{r}}\left(Z_{s}^{L}(\lambda)\right) \subseteq \mathcal{V}_{G_{r}}\left(Z_{s}(\lambda)\right)
$$

Our current assumption thus implies the projectivity of the module $\left.Z_{s}^{L}(\lambda)\right|_{L_{r}}$, see Proposition 1.1. Thanks to Corollary [5.5, this module belongs to a block $\mathcal{B} \subseteq \operatorname{Dist}\left(L_{r}\right) \cong \operatorname{Dist}\left(\operatorname{SL}(2)_{r}\right)^{r(\operatorname{dim} T-1)}$. If $\mathcal{B}$ is not a simple block, then [35, (II.11.16(b))] and [35, (II.11.10),(II.11.11)] in conjunction with $\mathrm{SL}(2)_{1}$-theory imply that the dimensions of the principal indecomposable $\mathcal{B}$-modules are even. As $\operatorname{dim}_{k} Z_{s}^{L}(\lambda)=p^{s}$, we have reached a contradiction. It follows that $\left.Z_{s}^{L}(\lambda)\right|_{L_{r}} \cong p^{s-r}\left(\mathrm{St}_{r} \otimes_{k} k_{\mu}\right)$, where $\mu \in X(T)$ satisfies $\left\langle\mu, \alpha^{\vee}\right\rangle=0$. Thus, $\left\langle\lambda+\rho, \alpha^{\vee}\right\rangle \in p^{r} \mathbb{Z}$, so that $\alpha \in \Psi_{\lambda}^{r}$. Since $\Psi_{\lambda}^{r}$ is a subsystem of $\Psi$ ([33, (2.7)]), we conclude that $\Psi=\Psi_{\lambda}^{r}$, as desired.

\section{Depth Reduction for Verma Modules}

Throughout this section, $G$ is assumed to be a connected reductive smooth algebraic group that is defined over the Galois field $\mathbb{F}_{p}$.

We fix $r \in \mathbb{N}$ and study non-projective Verma modules. Accordingly, we assume that $\lambda \in X(T)$ satisfies $\Psi \neq \Psi_{\lambda}^{r}$, that is, $\operatorname{dp}(\lambda) \leq r$. Recall that for any $d \in \mathbb{N}$ the Steinberg module $\mathrm{St}_{d} \cong$ $L\left(\left(p^{d}-1\right) \rho\right)$ has the structure of a $G$-module, so that it is also a $G_{r} T$-module, see [35, (II.10.1)]. Following [35, (II.3.16)], we denote by $M^{[d]}$ the $d$-Frobenius twist of a module $M$, defined over a subgroup scheme $H \subseteq G$. As before, the characteristic $p$ is assumed to be good for $G$. 
Proposition 6.1. Let $1 \leq d \leq r$. The Frobenius homomorphism $F^{d}: G_{r} \longrightarrow G_{r-d}$ induces an isomorphism $\mathcal{V}_{G_{r}}\left(\mathrm{St}_{d}\right) \stackrel{\sim}{\longrightarrow} \mathcal{V}_{G_{r-d}}(k)$ that maps $\mathcal{V}_{G_{r}}\left(M^{[d]} \otimes_{k} \mathrm{St}_{d}\right)$ onto $\mathcal{V}_{G_{r-d}}(M)$ for every $M \in$ $\bmod G_{r-d}$.

Proof. Given a $\operatorname{Dist}\left(G_{r}\right)$-module algebra $\Lambda$, we let $\mathrm{H}^{\bullet}\left(G_{r}, \Lambda\right):=\bigoplus_{n \geq 0} \mathrm{H}^{2 n}\left(G_{r}, \Lambda\right)$ be the corresponding even cohomology ring. The algebras we are interested in are of the form

$$
\begin{aligned}
\Lambda_{d}(M) & :=\operatorname{Hom}_{k}\left(M^{[d]} \otimes_{k} \mathrm{St}_{d}, M^{[d]} \otimes_{k} \mathrm{St}_{d}\right) \cong \operatorname{Hom}_{k}\left(M^{[d]}, M^{[d]}\right) \otimes_{k} \operatorname{Hom}_{k}\left(\mathrm{St}_{d}, \mathrm{St}_{d}\right) \\
& \cong \operatorname{Hom}_{k}(M, M)^{[d]} \otimes_{k} \operatorname{Hom}_{k}\left(\mathrm{St}_{d}, \mathrm{St}_{d}\right),
\end{aligned}
$$

where $M \in \bmod G_{r-d}$. Since $\Lambda_{d}(M)$ is a projective $G_{d^{-m o d u l e}}$, the Lyndon-Hochschild-Serre spectral sequence $\mathrm{H}^{n}\left(G_{r} / G_{d}, \mathrm{H}^{m}\left(G_{d}, \Lambda_{d}(M)\right)\right) \Rightarrow \mathrm{H}^{n+m}\left(G_{r}, \Lambda_{d}(M)\right)$, cf. [35, (I.6.6)], collapses to isomorphisms

$$
\mathrm{H}^{n}\left(G_{r} / G_{d}, \Lambda_{d}(M)^{G_{d}}\right) \cong \mathrm{H}^{n}\left(G_{r}, \Lambda_{d}(M)\right) \quad \forall n \geq 0 .
$$

In view of $\mathrm{St}_{d}=L\left(\left(p^{d}-1\right) \rho\right)$ being a simple $G$-module, the groups $G_{r}$ and $G_{r} / G_{d}$ operate trivially on the one-dimensional space $\operatorname{Hom}_{G_{d}}\left(\mathrm{St}_{d}, \mathrm{St}_{d}\right)$. As $G_{d}$ acts trivially on $\operatorname{Hom}_{k}(M, M)^{[d]}$, we obtain $G_{r}$-isomorphisms

$$
\Lambda_{d}(M)^{G_{d}} \cong \operatorname{Hom}_{k}(M, M)^{[d]} \otimes_{k} \operatorname{Hom}_{G_{d}}\left(\operatorname{St}_{d}, \operatorname{St}_{d}\right) \cong \operatorname{Hom}_{k}(M, M)^{[d]} .
$$

The canonical quotient map $\pi: G_{r} \longrightarrow G_{r} / G_{d}$ and the natural map $\operatorname{Hom}_{k}(M, M)^{[d]} \longrightarrow \Lambda_{d}(M)$; $f \mapsto f \otimes \mathrm{id}_{\mathrm{St}_{d}}$ induce homomorphisms

$$
\pi_{M}^{\bullet}: \mathrm{H}^{\bullet}\left(G_{r} / G_{d}, \operatorname{Hom}_{k}(M, M)^{[d]}\right) \longrightarrow \mathrm{H}^{\bullet}\left(G_{r}, \operatorname{Hom}_{k}(M, M)^{[d]}\right)
$$

and

$$
\Upsilon_{M}: \mathrm{H}^{\bullet}\left(G_{r}, \operatorname{Hom}_{k}(M, M)^{[d]}\right) \longrightarrow \mathrm{H}^{\bullet}\left(G_{r}, \Lambda_{d}(M)\right)
$$

of $k$-algebras, respectively. By the above, the inflation map $\Upsilon_{M} \circ \pi_{M}^{\bullet}$ is bijective (cf. [54, (6.7.3), (6.8.2)]). Thus, $\pi_{M}^{\bullet}$ is injective and, specializing $M=k$, we obtain $\mathrm{H}^{\bullet}\left(G_{r}, k\right)=\operatorname{im} \pi_{k}^{\bullet} \oplus \operatorname{ker} \Upsilon_{k}$. Consequently,

$$
\bar{\pi}_{k}^{\bullet}: \mathrm{H}^{\bullet}\left(G_{r} / G_{d}, k\right) \longrightarrow \mathrm{H}^{\bullet}\left(G_{r}, k\right) / \operatorname{ker} \Upsilon_{k} ; \quad x \mapsto \pi_{k}^{\bullet}(x)+\operatorname{ker} \Upsilon_{k}
$$

is an isomorphism of $k$-algebras. Thanks to [35, (I.9.5)], the Frobenius endomorphism $F^{d}$ induces an isomorphism $\omega_{d}: G_{r} / G_{d} \longrightarrow G_{r-d}$, so that

$$
\bar{\pi}_{k}^{\bullet} \circ \omega_{d}^{\bullet}: \mathrm{H}^{\bullet}\left(G_{r-d}, k\right) \longrightarrow \mathrm{H}^{\bullet}\left(G_{r}, k\right) / \operatorname{ker} \Upsilon_{k}
$$

is also an isomorphism. By definition, the variety $\mathcal{V}_{G_{r}}\left(\mathrm{St}_{d}\right)$ is the zero locus of the ideal ker $\Upsilon_{k}$, so that the associated comorphism is the desired isomorphism

$$
\mathcal{V}_{G_{r}}\left(\mathrm{St}_{d}\right) \stackrel{\sim}{\longrightarrow} \mathcal{V}_{G_{r-d}}(k) \text {. }
$$

(By construction, this isomorphism is the restriction of the morphism $\mathcal{V}_{G_{r}}(k) \longrightarrow \mathcal{V}_{G_{r-d}}(k)$ induced by $F^{d}$.) The Frobenius homomorphism $F^{d}$ gives rise to a commutative diagram

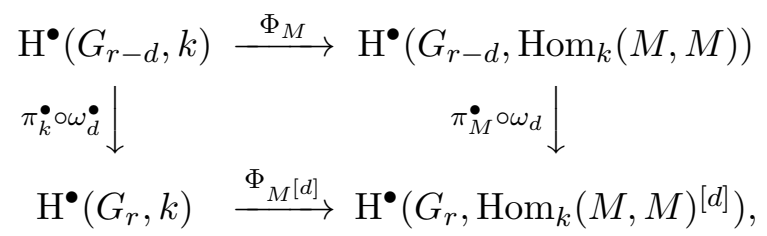

where the horizontal maps are given by $\alpha \mapsto \alpha . \operatorname{id}_{M}$. Since $\Upsilon_{M} \circ \pi_{M}^{\bullet}$ is bijective, we conclude the injectivity of the right-hand map. Owing to [51, (7.2)], we have

$$
\mathcal{V}_{G_{r}}\left(M^{[d]} \otimes_{k} \mathrm{St}_{d}\right)=\mathcal{V}_{G_{r}}\left(M^{[d]}\right) \cap \mathcal{V}_{G_{r}}\left(\mathrm{St}_{d}\right) \subseteq \mathcal{V}_{G_{r}}\left(\mathrm{St}_{d}\right)
$$


The intersection is given by the maximal ideal spectrum of $\mathrm{H}^{\bullet}\left(G_{r}, k\right) / I$, where $I=\operatorname{ker} \Phi_{M^{[d]}}+$ ker $\Upsilon_{k}$. From the above diagram, we conclude that the image of $I$ in $\mathrm{H}^{\bullet}\left(G_{r}, k\right) / \operatorname{ker} \Upsilon_{k}$ corresponds under the isomorphism $\bar{\pi}_{k}^{\bullet} \circ \omega_{d}^{\bullet}$ to the ideal ker $\Phi_{M}$. Consequently, the isomorphism $\mathcal{V}_{G_{r}}\left(\mathrm{St}_{d}\right) \stackrel{\sim}{\longrightarrow}$ $\mathcal{V}_{G_{r-d}}(k)$ induced by $\bar{\pi}_{k}^{\bullet} \circ \omega_{d}^{\bullet}$, sends $\mathcal{V}_{G_{r}}\left(M^{[d]}\right) \cap \mathcal{V}_{G_{r}}\left(\mathrm{St}_{d}\right)$ onto $\mathcal{V}_{G_{r-d}}(M)$, as desired.

Theorem 6.2. Suppose $G$ is semi-simple and simply connected and that $\lambda \in X(T)$ satisfies $r \geq$ $\operatorname{dp}(\lambda)=d+1 \geq 2$. Then there exists a weight $\mu \in X(T)$ of depth $\operatorname{dp}(\mu)=1$ such that

(a) there is an isomorphism $\widehat{Z}_{r}(\lambda) \cong \widehat{Z}_{r-d}(\mu)^{[d]} \otimes_{k} \mathrm{St}_{d}$, and

(b) there is an isomorphism $\mathcal{V}_{G_{r}}\left(Z_{r}(\lambda)\right) \cong \mathcal{V}_{G_{r-d}}\left(Z_{r-d}(\mu)\right)$.

Proof. Let $e_{d} \in \operatorname{Dist}\left(G_{d}\right) \subseteq \operatorname{Dist}\left(G_{r}\right)$ be the central primitive idempotent, defined by the projective simple $G_{d}$-module $\mathrm{St}_{d}$. Owing to [35, (II.10.3)], $e_{d}$ is a central idempotent of $\operatorname{Dist}\left(G_{r}\right)$. Since $\operatorname{dp}(\lambda)=d+1$, we have $\Psi_{\lambda}^{d}=\Psi$. As $G$ is semi-simple and simply connected, $\mathrm{St}_{d}$ is the only simple, projective $G_{r}$-module. Accordingly, Corollary 5.6 provides an isomorphism $\left.Z_{r}(\lambda)\right|_{G_{d}} \cong n \mathrm{St}_{d}$, so

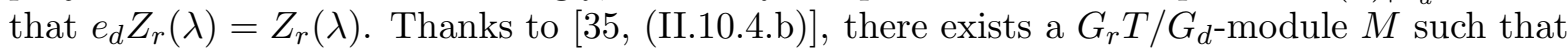

$$
Z_{r}(\lambda) \cong M \otimes_{k} \mathrm{St}_{d}
$$

In particular, $\operatorname{dim}_{k} M=p^{(r-d) \operatorname{dim}_{k} \mathfrak{u}}$.

Setting $H=G_{d} B_{r}^{-}$, we propose to show that $Z_{r}(\lambda)$ is a projective $H$-module. Recall that $V_{r}(H)_{Z_{r}(\lambda)} \subseteq V_{r}(G)_{Z_{r}(\lambda)} \subseteq V_{r}(U)$, whence

$$
V_{r}(H)_{Z_{r}(\lambda)}=V_{r}\left(H \cap U_{r}\right)_{Z_{r}(\lambda)}
$$

Let $R$ be a commutative $k$-algebra and let $x$ be an element of $\left(H \cap U_{r}\right)(R)$. By general theory [53. (15.5)], there exists a faithfully flat extension $S$ of $R$ such that $x_{S}=a b$ for $a \in G_{d}(S)$ and $b \in B_{r}^{-}(S)$. Here $x_{S}$ denotes the image of $x$ in $\left(H \cap U_{r}\right)(S)$ under the canonical map induced by $R \longrightarrow S$. An application of the $d$-th power of the Frobenius endomorphism gives

$$
F^{d}(x)_{S}=F^{d}\left(x_{S}\right)=F^{d}(a) F^{d}(b)=F^{d}(b) \in U_{r-d}(S) \cap B_{r-d}^{-}(S)=\{1\} .
$$

In view of [53, (15.6)], we also have $F^{d}(x)=1$, whence $x \in U_{d}(R)$. Thus, $H \cap U_{r} \subseteq U_{d}$ implying

$$
V_{r}\left(H \cap U_{r}\right)_{Z_{r}(\lambda)} \subseteq V_{r}\left(U_{d}\right)_{Z_{r}(\lambda)} \subseteq V_{r}\left(G_{d}\right)_{Z_{r}(\lambda)} \cong V_{d}\left(G_{d}\right)_{Z_{r}(\lambda)},
$$

where the last isomorphism is obtained by restricting the canonical identification $V_{d}\left(G_{d}\right) \longrightarrow$ $V_{r}\left(G_{d}\right) ; \varphi \mapsto \varphi \circ F^{r-d}$. In view of Corollary [5.6, the right-hand variety is trivial, proving that $V_{r}(H)_{Z_{r}(\lambda)}=V_{r}\left(H \cap U_{r}\right)_{Z_{r}(\lambda)}=\{0\}$. As a result, the module $\left.Z_{r}(\lambda)\right|_{H}$ is projective.

Owing to [35, (I.9.5)], the Frobenius homomorphism $F^{d}$ induces an isomorphism $G_{r} T / G_{d} \cong$ $G_{r-d} T$. Consequently, $M=N^{[d]}$ for some $G_{r-d} T$-module $N$. Moreover, $N$ is a projective $B_{r-d^{-}}^{-}$ module if and only if $M$ is a projective $H / G_{d}$-module. By [35. (II.10.4)], the functor $X \mapsto X \otimes_{k} \mathrm{St}_{d}$ gives rise to an equivalence between $\bmod \operatorname{Dist}\left(H / G_{d}\right)$ and $\bmod \operatorname{Dist}(H) e_{d}$, sending $M$ to $Z_{r}(\lambda)$. Since $Z_{r}(\lambda)$ is a projective $H$-module, we conclude the $B_{r-d^{-}}^{-}$-projectivity of $N$. According to [35, (II.11.2)], the $G_{r-d} T$-module $N$ therefore affords a filtration by baby Verma modules. Thus, for dimension reasons, we have $N \cong Z_{r-d}(\nu)$ for some $\nu \in X(T)$.

Since

$$
\mathfrak{F}\left(\widehat{Z}_{r-d}(\nu)^{[d]} \otimes_{k} \mathrm{St}_{d}\right) \cong \mathfrak{F}\left(\widehat{Z}_{r-d}(\nu)^{[d]}\right) \otimes_{k} \mathfrak{F}\left(\mathrm{St}_{d}\right) \cong Z_{r-d}(\nu)^{[d]} \otimes_{k} \mathrm{St}_{d} \cong Z_{r}(\lambda),
$$

it follows from [26, (4.1)] that there exists $\gamma \in X(T)$ such that

$$
\widehat{Z}_{r}(\lambda) \cong \widehat{Z}_{r-d}(\nu)^{[d]} \otimes_{k} \mathrm{St}_{d} \otimes_{k} k_{p^{r} \gamma} \cong \widehat{Z}_{r-d}\left(\nu+p^{r-d} \gamma\right)^{[d]} \otimes_{k} \mathrm{St}_{d} .
$$

A comparison of highest weight vectors gives $\lambda=p^{d} \nu+p^{r} \gamma+\left(p^{d}-1\right) \rho$. Consequently,

$$
\left\langle\lambda+\rho, \alpha^{\vee}\right\rangle=p^{d}\left\langle\nu+p^{r-d} \gamma+\rho, \alpha^{\vee}\right\rangle
$$


for all $\alpha \in \Psi$, so that the weight $\mu:=\nu+p^{r-d} \gamma$ has depth $\operatorname{dp}(\mu)=1$. This completes the proof of (a). Part (b) is now a direct consequence of Proposition 6.1.

Example. We consider the semi-simple, simply connected group $G=\mathrm{SL}(2)$. If $\lambda \in X(T)$ is a weight with $\operatorname{dp}(\lambda) \leq r$, then

$$
\mathcal{V}_{\mathrm{SL}(2)_{r}}\left(Z_{r}(\lambda)\right) \cong k^{r+1-\operatorname{dp}(\lambda)}
$$

If $\operatorname{dp}(\lambda)=1$, then our assertion follows directly from Corollary 5.2. Alternatively, Theorem 6.2 reduces us to this case.

Corollary 6.3. Let $p \geq h$ and suppose that $G$ is semi-simple and simply connected. Let $\lambda \in$ $X(T)$ be a weight of depth $\operatorname{dp}(\lambda) \leq r$ such that $\mathcal{V}_{U_{r+1-\mathrm{dp}(\lambda)}}(k)$ is irreducible. Then $\mathcal{V}_{G_{r}}\left(Z_{r}(\lambda)\right) \cong$ $\mathcal{V}_{U_{r+1-\operatorname{dp}(\lambda)}}(k)$ if and only if $\lambda$ is $p^{\mathrm{dp}(\lambda)}$-regular.

Proof. Thanks to Theorem 6.2, there exists a weight $\mu \in X(T)$ of depth 1 and isomorphisms

$$
Z_{r}(\lambda) \cong Z_{r-d}(\mu)^{[d]} \otimes_{k} \operatorname{St}_{d} \text { and } \mathcal{V}_{G_{r}}\left(Z_{r}(\lambda)\right) \cong \mathcal{V}_{G_{r-d}}\left(Z_{r-d}(\mu)\right)
$$

of modules and varieties, respectively. According to Lemma 5.1, the condition $\mathcal{V}_{G_{r-d}}\left(Z_{r-d}(\mu)\right) \cong$ $\mathcal{V}_{U_{r-d}}(k)$ is equivalent to $\mu$ being $p$-regular. Since $\lambda=p^{d} \mu+\left(p^{d}-1\right) \rho$, this happens precisely when $\lambda$ is $p^{\mathrm{dp}(\lambda)}$-regular.

Example. Consider the semi-simple, simply connected group $G=\mathrm{SL}(3)$. If $\lambda \in X(T)$ has depth $\operatorname{dp}(\lambda) \leq r$ and $\lambda$ is $p^{\mathrm{dp}(\lambda)}$-regular, then the example following Lemma 5.1 in conjunction with Corollary 6.3 implies $\mathcal{V}_{\mathrm{SL}(3)_{r}}\left(Z_{r}(\lambda)\right) \cong \mathcal{V}_{U_{r+1-\mathrm{dp}(\lambda)}}(k)$ as well as $\operatorname{cx}_{\mathrm{SL}(3)_{r}}\left(Z_{r}(\lambda)\right)=2(r-\mathrm{dp}(\lambda))+3$.

Corollary 6.4. Let $p \geq h$ and suppose that $G$ is semi-simple and simply connected. Let $\lambda \in X(T)$ be a weight of depth $\operatorname{dp}(\lambda)=r$. Then $\mathcal{V}_{G_{r}}\left(Z_{r}(\lambda)\right) \cong \mathcal{V}_{U_{1}}(k)$ if and only if $\lambda$ is $p^{r}$-regular.

Corollary 6.5. Suppose that $G$ is semi-simple, simply connected and of characteristic $p \geq 5$. Let $\lambda \in X(T)$ be a weight such that $\operatorname{cx}_{G_{r}}\left(Z_{r}(\lambda)\right)=r+1-\operatorname{dp}(\lambda)$. Then there exists a simple root $\alpha \in \Psi$ such that $\mathcal{V}_{G_{r}}\left(Z_{r}(\lambda)\right) \cong \mathcal{V}_{\left(U_{\alpha}\right)_{r+1-\operatorname{dp}(\lambda)}}(k)$ is irreducible.

Proof. As before, we write $\operatorname{dp}(\lambda)=d+1$. If $\operatorname{dp}(\lambda)=1$, then the result follows directly from Corollary [5.3. Alternatively, Theorem 6.2(b) provides a weight $\mu$ of depth 1 and an isomorphism $\mathcal{V}_{G_{r}}\left(Z_{r}(\lambda)\right) \cong \mathcal{V}_{G_{r-d}}\left(Z_{r-d}(\mu)\right)$, so that another application of Corollary 5.3 completes the proof.

The following result reduces questions concerning AR-components of Verma modules to those, whose defining weights have depth 1 . For ease of notation, we shall often denote Verma modules defined over different groups by the same symbol. 
Theorem 6.6. Let $\lambda \in X(T)$ be a weight such that $r \geq \operatorname{dp}(\lambda)=d+1 \geq 1$. Then there exists a covering group $\tilde{G}$ of $G$ and functors

$$
\Phi: \bmod G_{r} T \longrightarrow \bmod \tilde{G}_{r-d} \tilde{T} \text { and } \Upsilon: \bmod G_{r} \longrightarrow \bmod \tilde{G}_{r-d}
$$

with the following properties:

(a) The diagram

commutes.

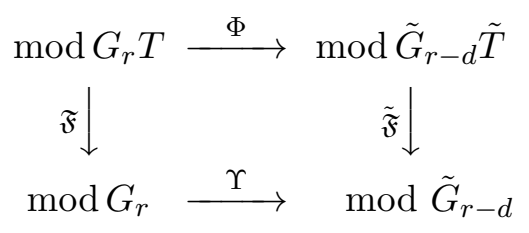

(b) There is a weight $\mu \in X(\tilde{T})$ of depth $\operatorname{dp}(\mu)=1$ such that $\Phi\left(\widehat{Z}_{r}(\lambda)\right) \cong \widehat{Z}_{r-d}(\mu)$.

(c) We have $\operatorname{cx}_{G_{r}}\left(\widehat{Z}_{r}(\lambda)\right)=\operatorname{cx}_{\tilde{G}_{r-d}}\left(\widehat{Z}_{r-d}(\mu)\right) \geq r-d$.

(d) The functor $\Phi$ induces an isomorphism $\widehat{\Theta}_{r}(\lambda) \longrightarrow \widehat{\Theta}_{r-d}(\mu) ;[M] \mapsto[\Phi(M)]$ of stable translation quivers.

Proof. In view of Corollary [5.2, our statement holds for $d=0$. We therefore assume that $d \geq 1$.

Proceeding in several steps, we first prove our result under the assumption:

(i) $G$ is semi-simple and simply connected.

Owing to Theorem 6.2, there exists a weight $\mu \in X(T)$ such that $\operatorname{dp}(\mu)=1$ and

$$
\widehat{Z}_{r}(\lambda) \cong \widehat{Z}_{r-d}(\mu)^{[d]} \otimes_{k} \mathrm{St}_{d}
$$

Letting $e_{d} \in \operatorname{Dist}\left(G_{d}\right) \subseteq \operatorname{Dist}\left(G_{r}\right)$ be the primitive idempotent belonging to $\mathrm{St}_{d}$, we denote by $\bmod G_{r} T e_{d}$ the full subcategory of $\bmod G_{r} T$, consisting of those $G_{r} T$-modules $M$ satisfying $e_{d} M=$ $M$. In view of [35, (II.10.4)], $\bmod G_{r} T e_{d}$ is a sum of blocks of $\bmod G_{r} T$.

Since the Frobenius endomorphism $F^{d}$ induces an isomorphism $G_{r} T / G_{d} \cong G_{r-d} T$, an application of [35, (II.10.4/5)] shows that the restriction of the functor

$$
\Phi: \bmod G_{r} T \longrightarrow \bmod G_{r-d} T \quad ; \quad M \mapsto \operatorname{Hom}_{G_{d}}\left(\mathrm{St}_{d}, M\right)^{[-d]}
$$

to $\bmod G_{r} T e_{d}$ is an equivalence of categories. As such, $\Phi$ induces an isomorphism between the stable Auslander-Reiten quivers $\Gamma_{s}\left(\bmod G_{r} T e_{d}\right)$ and $\Gamma_{s}\left(G_{r-d} T\right)$, sending the class of the module $\widehat{Z}_{r}(\lambda) \in \bmod G_{r} T e_{d}$ onto that of $\widehat{Z}_{r-d}(\mu)$. Since $\bmod G_{r} T e_{d}$ is a sum of blocks of $\bmod G_{r} T$, the component of $\Gamma_{s}\left(\bmod G_{r} T e_{d}\right)$ containing $\left[\widehat{Z}_{r}(\lambda)\right]$ coincides with $\widehat{\Theta}_{r}(\lambda)$. Consequently, $\Phi\left(\widehat{\Theta}_{r}(\lambda)\right)=$ $\widehat{\Theta}_{r-d}(\mu)$, so that (b) and (d) hold for $\tilde{G}:=G$.

Another application of [35, (II.10.4/5)] shows that the restriction of

$$
\Upsilon: \bmod G_{r} \longrightarrow \bmod G_{r-d} \quad ; \quad M \mapsto \operatorname{Hom}_{G_{d}}\left(\mathrm{St}_{d}, M\right)^{[-d]},
$$

to $\bmod \operatorname{Dist}\left(G_{r}\right) e_{d}$ is an equivalence of categories. By definition, we have $\mathfrak{F} \circ \Phi=\Upsilon \circ \mathfrak{F}$. Since $\Psi_{\mu} \neq \Psi$, a consecutive application of Corollary 5.2 and Theorem 6.2 yields

$$
r-d \leq \operatorname{cx}_{G_{r-d}}\left(\widehat{Z}_{r-d}(\mu)\right)=\operatorname{cx}_{G_{r}}\left(\widehat{Z}_{r}(\lambda)\right),
$$

as desired.

(ii) $G$ is semi-simple.

We consider a semi-simple simply connected covering group $\tilde{G}$ along with the maps $\pi: \tilde{G}_{r} \tilde{T} \longrightarrow G_{r} T$ and $\sigma: \tilde{G}_{r} \longrightarrow G_{r}$ that are induced by the canonical quotient map $\omega: \tilde{G} \longrightarrow G$. By Lemma 3.5 , the corresponding pull-back functor $\pi^{*}: \bmod G_{r} T \longrightarrow \bmod \tilde{G}_{r} \tilde{T}$ allows us to view $\bmod G_{r} T$ as the sum of those blocks of $\bmod \tilde{G}_{r} \tilde{T}$, whose modules have weights belonging to $X(T) \subseteq X(\tilde{T})$. 
We first show that the pull-back $\pi^{*}\left(\widehat{Z}_{r}(\lambda)\right)$ coincides with the baby Verma module $\widehat{\tilde{Z}}_{r}(\lambda)$ of $\bmod \tilde{G}_{r} \tilde{T}$. Since $\tilde{G}$ is a covering, the map $\omega$ induces an isomorphism $\tilde{U}^{-} \longrightarrow U^{-}$, cf. [35, (II.9.7)]. Consequently, $\pi^{*}\left(\widehat{Z}_{r}(\lambda)\right)$ is a $\tilde{U}_{r}^{-}$-projective $\tilde{G}_{r} \tilde{T}$-module. In view of the isomorphism $\tilde{B}_{r}^{-} \cong \tilde{U}_{r}^{-} \rtimes \tilde{T}_{r}$, our module is even $\tilde{B}_{r}^{-}$-projective. Thus, [35, (II.11.2)] provides a filtration of $\pi^{*}\left(\widehat{Z}_{r}(\lambda)\right)$ by baby Verma modules, which, for dimension reasons, only has one constituent $\widehat{\tilde{Z}}_{r}(\mu)$. Since all weights of $\pi^{*}\left(\widehat{Z}_{r}(\lambda)\right)$ are $\leq \lambda$, while all weights of $\widehat{\tilde{Z}}_{r}(\mu)$ are $\leq \mu$, we obtain $\lambda=\mu$.

The depth of $\lambda \in X(T)$ coincides with the depth of $\lambda$, viewed as an element of $X(\tilde{T})$ (see 35, (II.1.17)]). By part (i), we have functors $\Phi_{1}: \bmod \tilde{G}_{r} \tilde{T} \longrightarrow \bmod \tilde{G}_{r-d} \tilde{T}$ and $\Upsilon_{1}: \bmod \tilde{G}_{r} \longrightarrow$ $\bmod \tilde{G}_{r-d}$ satisfying (a)-(c). In particular, there exists a weight $\mu \in X(\tilde{T})$ of depth 1 such that

$$
\Phi_{1}\left(\widehat{\tilde{Z}}_{r}(\lambda)\right) \cong \widehat{\tilde{Z}}_{r-d}(\mu) .
$$

We now consider the functors $\Phi_{2}:=\Phi_{1} \circ \pi^{*}$ and $\Upsilon_{2}:=\Upsilon_{1} \circ \sigma^{*}$. We thus have $\tilde{\mathfrak{F}} \circ \pi^{*}=\sigma^{*} \circ \mathfrak{F}$, whence

$$
\tilde{\mathfrak{F}} \circ \Phi_{2}=\Upsilon_{1} \circ \tilde{\mathfrak{F}} \circ \pi^{*}=\Upsilon_{1} \circ \sigma^{*} \circ \mathfrak{F}=\Upsilon_{2} \circ \mathfrak{F}
$$

Moreover, there are isomorphisms

$$
\Phi_{2}\left(\widehat{Z}_{r}(\lambda)\right) \cong \Phi_{1}\left(\pi^{*}\left(\widehat{Z}_{r}(\lambda)\right)\right) \cong \Phi_{1}\left(\widehat{\tilde{Z}}_{r}(\lambda)\right) \cong \widehat{\tilde{Z}}_{r-d}(\mu),
$$

so that (a) and (b) hold.

In view of (i), property (c) follows from the equality $\operatorname{cx}_{\tilde{G}_{r}}\left(\pi^{*}\left(\widehat{Z}_{r}(\lambda)\right)\right)=\operatorname{cx}_{G_{r}}\left(\widehat{Z}_{r}(\lambda)\right)$, which can be seen as follows: Let $\left(\widehat{P}_{n}\right)_{n \geq 0}$ be a minimal projective resolution of the $G_{r} T$-module $\widehat{Z}_{r}(\lambda)$. Owing to Lemma 3.5, $\left(\pi^{*}\left(\widehat{P}_{n}\right)\right)_{n \geq 0}$ is a minimal projective resolution of $\pi^{*}\left(\widehat{Z}_{r}(\lambda)\right)$. Since $\operatorname{Top}_{G_{r}}(\mathfrak{F}(M))=$ $\mathfrak{F}\left(\operatorname{Top}_{G_{r} T}(M)\right)$ for every $G_{r} T$-module $M$ (see [35, (II.9.6.(11))]), the forgetful functor $\mathfrak{F}$ takes projective covers to projective covers (cf. also [27, (1.3)] and [34, (1.6)]). Consequently, the complexities $\operatorname{cx}_{G_{r}}\left(\widehat{Z}_{r}(\lambda)\right)$ and $\operatorname{cx}_{\tilde{G}_{r}}\left(\pi^{*}\left(\widehat{Z}_{r}(\lambda)\right)\right)$ are computable from the growths of the sequences $\left(\operatorname{dim}_{k} \widehat{P}_{n}\right)_{n \geq 0}=\left(\operatorname{dim}_{k} \pi^{*}\left(\widehat{P}_{n}\right)\right)_{n \geq 0}$.

By virtue of Lemma 3.5, the functor $\pi^{*}$ induces an isomorphism

$$
\widehat{\Theta}_{r}(\lambda) \longrightarrow \widehat{\tilde{\Theta}}_{r}(\lambda) ;[M] \mapsto\left[\pi^{*}(M)\right],
$$

so that $\Phi_{2}$ enjoys the required property $(\mathrm{d})$.

(iii) $G=G^{\prime} \times T^{\prime \prime}$, with a semi-simple group $G^{\prime}$ and a torus $T^{\prime \prime}$.

We denote by $T^{\prime}$ a maximal torus of $G^{\prime}$, so that $T:=T^{\prime} \times T^{\prime \prime}$ is a maximal torus of $G$. Then $X(T) \cong X\left(T^{\prime}\right) \times X\left(T^{\prime \prime}\right)$, and our weight $\lambda$ has the form

$$
\lambda=\left(\lambda^{\prime}, \lambda^{\prime \prime}\right),
$$

where $\operatorname{dp}\left(\lambda^{\prime}\right)=\operatorname{dp}(\lambda)$. According to step (ii), we can find a covering $\tilde{G}^{\prime} \longrightarrow G^{\prime}$ and functors

$$
\Phi_{2}: \bmod G_{r}^{\prime} T^{\prime} \longrightarrow \bmod \tilde{G}_{r-d}^{\prime} \tilde{T}^{\prime} \text { and } \Upsilon_{2}: \bmod G_{r}^{\prime} \longrightarrow \bmod \tilde{G}_{r-d}^{\prime}
$$

as well as a weight $\mu^{\prime} \in X\left(\tilde{T}^{\prime}\right)$ of depth 1 such that (a)-(d) hold.

Let $\tilde{G}:=\tilde{G}^{\prime} \times T^{\prime \prime}$, so that $\tilde{G}$ is a covering of $G$. Since $\bmod G_{r} T$ is a sum of copies of $\bmod G_{r}^{\prime} T^{\prime}$, with each constituent being given by those $G_{r} T$-modules, whose weights belong to $X\left(T^{\prime}\right) \times\{\gamma\}$ for a fixed $\gamma \in X\left(T^{\prime \prime}\right)$, we consider the composite

$$
\Phi_{3}:=\iota_{\lambda^{\prime \prime}} \circ \Phi_{2} \circ \mathrm{pr}_{\lambda^{\prime \prime}}
$$

where

$$
\iota_{\lambda^{\prime \prime}}: \bmod \tilde{G}_{r-d}^{\prime} \tilde{T}^{\prime} \longrightarrow \bmod \tilde{G}_{r-d} \tilde{T} \text { and } \operatorname{pr}_{\lambda^{\prime \prime}}: \bmod G_{r} T \longrightarrow \bmod G_{r}^{\prime} T^{\prime}
$$


are the inclusion functor and the projection functor induced by the decompositions of the relevant categories. Note that $\operatorname{pr}_{\lambda^{\prime \prime}}$ sends $\widehat{Z}_{r}(\lambda)$ to $\widehat{Z}_{r}\left(\lambda^{\prime}\right)$, so that

$$
\Phi_{3}\left(\widehat{Z}_{r}(\lambda)\right) \cong \widehat{Z}_{r-d}\left(\mu^{\prime}, \lambda^{\prime \prime}\right) \text {. }
$$

As noted earlier, the weight $\mu:=\left(\mu^{\prime}, \lambda^{\prime \prime}\right)$ satisfies $\operatorname{dp}(\mu)=\operatorname{dp}\left(\mu^{\prime}\right)=1$. Since the elements of $\widehat{\Theta}_{r}(\lambda)$ and $\widehat{\Theta}_{r-d}(\mu)$ belong to the respective blocks defined by $\lambda^{\prime \prime}$, the functors $\iota_{\lambda^{\prime \prime}}$ and $\operatorname{pr}_{\lambda^{\prime \prime}}$ give rise to isomorphisms $\widehat{\Theta}_{r-d}\left(\mu^{\prime}\right) \longrightarrow \widehat{\Theta}_{r-d}(\mu)$ and $\widehat{\Theta}_{r}(\lambda) \longrightarrow \Theta_{r}\left(\lambda^{\prime}\right)$ of stable translation quivers. Consequently, $\Phi_{3}$ satisfies (d).

The module category $\bmod G_{r}$ has a decomposition that is compatible with the one given above. Hence the definition

$$
\Upsilon_{3}:=\iota_{\lambda^{\prime \prime}} \circ \Upsilon_{2} \circ \mathrm{pr}_{\lambda^{\prime \prime}}
$$

yields a functor so that the pair $\left(\Phi_{3}, \Upsilon_{3}\right)$ satisfies (a).

The block decompositions give rise to

$$
\operatorname{cx}_{G_{r}}\left(\widehat{Z}_{r}(\lambda)\right)=\operatorname{cx}_{G_{r}^{\prime}}\left(\widehat{Z}_{r}\left(\lambda^{\prime}\right)\right) \text { and } \operatorname{cx}_{\tilde{G}_{r-d}}\left(\widehat{Z}_{r-d}(\mu)\right)=\operatorname{cx}_{\tilde{G}_{r-d}^{\prime}}\left(\widehat{Z}_{r-d}\left(\mu^{\prime}\right)\right)
$$

so that (c) follows from (ii).

(iv) $G$ is reductive.

General theory provides a covering $(G, G) \times Z(G)^{\circ} \stackrel{\omega}{\longrightarrow} G$, with the group $G^{\prime}:=(G, G) \times Z(G)^{\circ}$ being of the type discussed in (iii). Let $\tilde{G}^{\prime}$ be a covering of $G^{\prime}$ satisfying (a)-(d). Arguing as in step (ii), we obtain the desired functors by combining those of (iii) with the pull-back functors defined by $\omega$. Since the kernel of the canonical map $\tilde{G}^{\prime} \longrightarrow G$ is a normal subgroup of dimension zero, it is contained in the center $Z\left(\tilde{G}^{\prime}\right)$ and thus in particular, it is abelian. As it also is an extension of two diagonalizable group schemes, [12, (IV.§1.(4.5))] ensures that it is diagonalizable. Consequently, the group $\tilde{G}^{\prime}$ is indeed a covering of $G$.

For our next result, recall the notation $x^{+}$from Lemma 3.3 for the set of successors of a vertex $x$ in the stable AR-quiver $\Gamma_{s}\left(G_{r}\right)$.

Corollary 6.7. Let $\lambda \in X(T)$ be a weight such that $r \geq \operatorname{dp}(\lambda)=d+1 \geq 1$. Then there exists a covering group $\tilde{G}$ and a weight $\mu \in X(\tilde{T})$ with the following properties.

(a) There exists a surjective morphism $\Upsilon: \Theta_{r}(\lambda) \longrightarrow \Theta_{r-d}(\mu)$ of stable translation quivers such that $\Upsilon\left(x^{+}\right)=\Upsilon(x)^{+}$for all $x \in \Theta_{r}(\lambda)$.

(b) If $G$ is semi-simple and simply connected, then $\Upsilon$ is an isomorphism.

Proof. According to Theorem 6.6, there exists a covering group $\tilde{G}$ and functors

$$
\Phi: \bmod G_{r} T \longrightarrow \bmod \tilde{G}_{r-d} \tilde{T} \text { and } \Upsilon: \bmod G_{r} \longrightarrow \bmod \tilde{G}_{r-d}
$$

giving rise to a commutative diagram

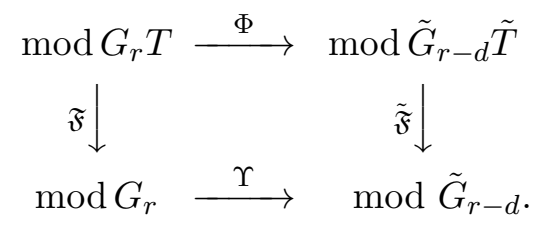

In addition, the functor $\Phi$ induces an isomorphism

$$
\widehat{\Theta}_{r}(\lambda) \longrightarrow \widehat{\Theta}_{r-d}(\mu) ;[M] \mapsto[\Phi(M)]
$$

for a suitable weight $\mu \in X(\tilde{T})$ of depth 1 . In view of Lemma 3.3 and the above diagram, the map

$$
\Upsilon: \Theta_{r}(\lambda) \longrightarrow \Theta_{r-d}(\mu) ;[M] \mapsto[\Upsilon(M)]
$$


is well-defined.

If $\alpha: x \rightarrow y$ is an arrow in $\Theta_{r}(\lambda)$, then there exists an arrow $\hat{\alpha}: \hat{x} \rightarrow \hat{y}$ in $\widehat{\Theta}_{r}(\lambda)$ such that $\mathfrak{F}(\hat{x})=x$ and $\mathfrak{F}(\hat{y})=y$. Consequently, $\Upsilon(\alpha):=\tilde{\mathfrak{F}}(\Theta(\hat{\alpha}))$ is an arrow in $\Theta_{r-d}(\mu)$ with starting point $\tilde{\mathfrak{F}}(\Phi(\hat{x}))=\Upsilon(\mathfrak{F}(\hat{x}))=\Upsilon(x)$ and endpoint $\tilde{\mathfrak{F}}(\Phi(\hat{y}))=\Upsilon(y)$. With this definition, $\Upsilon$ becomes a morphism of quivers. Since $\mathfrak{F}, \Phi$, and $\tilde{\mathfrak{F}}$ commute with translations, so does $\Upsilon$. Finally, given $x=\mathfrak{F}(\hat{x}) \in \Theta_{r}(\lambda)$, we obtain, observing Lemma 3.3,

$$
\Upsilon\left(x^{+}\right)=\Upsilon\left(\mathfrak{F}\left(\hat{x}^{+}\right)\right)=\tilde{\mathfrak{F}}\left(\Phi\left(\hat{x}^{+}\right)\right)=\tilde{\mathfrak{F}}\left(\Phi(\hat{x})^{+}\right)=(\tilde{\mathfrak{F}} \circ \Phi)(\hat{x})^{+}=\Upsilon(x)^{+},
$$

as desired.

Property (b) follows directly from step (i) of the proof of Theorem 6.6

Example. Let $G=\mathrm{SL}(2)$ and $\lambda=p^{r-1} a-1, a \notin p \mathbb{Z}$ be a weight of depth $r$. If $r \geq 2$, then Corollary 6.7 implies $\Theta_{r}(\lambda) \cong \Theta_{1}(a-1)$. Since $\Theta_{1}(a-1)$ has a one-dimensional support variety, it is of the form $\mathbb{Z}\left[A_{\infty}\right] /(\tau)$, with $Z_{1}(a-1)$ having exactly one predecessor. Consequently, $\Theta_{r}(\lambda)$ and $Z_{r}(\lambda)$ have the same properties.

\section{Verma Modules of Complexity at most 2}

The purpose of this final section is to indicate the utility of the results and methods established so far. In particular, we show that questions concerning Verma modules of complexity at most 2 can be reduced to the case where the underlying groups are the first and second Frobenius kernels of SL(2). Throughout, $G$ is assumed to be defined over $\mathbb{F}_{p}$.

We begin with the following refinement of Lemma 4.1.

Lemma 7.1. Suppose that $G$ is semi-simple, simply connected and that $p$ is good for $G$. Let $Z_{r}(\lambda)$ be a Verma module of complexity 1 . Then $\Theta_{r}(\lambda) \cong \mathbb{Z}\left[A_{\infty}\right] /(\tau)$ is a homogeneous tube.

Proof. Writing $d+1=\operatorname{dp}(\lambda)$, we obtain from Theorem 6.6 the identity $r-d=1$. Thus, Corollary 6.7 provides an isomorphism

$$
\Theta_{r}(\lambda) \cong \Theta_{1}(\mu)
$$

for some weight $\mu$. In particular, the component $\Theta_{1}(\mu)$ consists of $\tau$-periodic vertices, and [15, (2.5)] yields the result.

As a first Corollary, we show that Verma modules of complexity 2 possess equidimenisonal support varieties:

Corollary 7.2. Let $G$ be semi-simple and simply connected. Suppose that $p \geq 7$, and let $\lambda \in X(T)$ be a weight such that $\mathrm{cx}_{G_{r}}\left(Z_{r}(\lambda)\right)=2$. Then $\mathcal{V}_{G_{r}}\left(Z_{r}(\lambda)\right)$ is equidimensional.

Proof. In view of Theorem 6.2, we may assume that $\operatorname{dp}(\lambda)=1$. Thanks to Corollary 5.2, we thus have

$$
2=\operatorname{cx}_{G_{r}}\left(Z_{r}(\lambda)\right) \geq r
$$

and Corollary 6.5 yields the irreducibility of $\mathcal{V}_{G_{r}}\left(Z_{r}(\lambda)\right)$ in case $r=2$. Alternatively, [22, (3.3)] implies that the rank variety $V_{1}(G)_{Z_{1}(\lambda)}$ is equidimenional. Owing to [24, (1.4)], the support variety $\mathcal{V}_{G_{1}}\left(Z_{1}(\lambda)\right)$ enjoys the same property. 
By showing that Verma modules of complexity 2 over higher Frobenius kernels essentially only occur for $\mathrm{SL}(2)_{2}$, our next result extends [22, (3.5)]. We leave the modifications concerning modules of complexity 1 to the interested reader.

Proposition 7.3. Suppose $r \geq 2, p \geq 7$, and let $\lambda \in X(T)$ be a weight such that $\operatorname{dp}(\lambda)=1$ and $\operatorname{cx}_{G_{r}}\left(Z_{r}(\lambda)\right) \leq 2$. Then the following statements hold:

(1) $r=2$ and $G_{r} \cong S_{r} \times H_{r}$, where $S \cong \mathrm{SL}(2)$ and $H$ is reductive.

(2) There is an isomorphism $Z_{r}(\lambda) \cong Z_{r}^{S}(\lambda) \otimes_{k} Z_{r}^{H}(\lambda)$, with $\operatorname{cx}_{S_{r}}\left(Z_{r}^{S}(\lambda)\right)=\operatorname{cx}_{G_{r}}\left(Z_{r}(\lambda)\right)$, and the second factor being a simple projective $H_{r}$-module.

Proof. Since $\Psi_{\lambda} \neq \Psi$ and $p$ is good, there exists a simple root $\alpha$ in $\Psi \backslash \Psi_{\lambda}$. According to Corollary 5.2. we have $r=2$ as well as $\mathcal{V}_{\left(U_{\alpha}\right)_{r}}(k) \subseteq \mathcal{V}_{G_{r}}\left(Z_{r}(\lambda)\right)$. In view of [50, (1.14)] and [51, (5.2),(6.8)], we obtain the same inclusion for the corresponding rank varieties:

$$
V_{r}\left(U_{\alpha}\right) \subseteq V_{r}(G)_{Z_{r}(\lambda)} .
$$

Thus, $V_{r}\left(U_{\alpha}\right) \cong V_{r}\left(\mathbb{G}_{a}\right) \cong k^{r}$ (cf. [50, (1.10)]) is an irreducible component of the $B$-invariant variety $V_{r}(G)_{Z_{r}(\lambda)}$. Hence $V_{r}\left(U_{\alpha}\right)$ is $B$-invariant, and the orbit $B . \varphi$ of the canonical isomorphism $\mathbb{G}_{a(r)} \stackrel{\varphi}{\longrightarrow}\left(U_{\alpha}\right)_{r}$ belongs to $V_{r}\left(U_{\alpha}\right)$. This implies that $\left(U_{\alpha}\right)_{r}$ is $B$-invariant, so that its Lie algebra $\operatorname{Lie}\left(\left(U_{\alpha}\right)_{r}\right)=\operatorname{Lie}\left(U_{\alpha}\right)=\mathfrak{g}_{\alpha}$ enjoys the same property. An application of [22, (3.3)] (and its proof) now implies that $G=S H$ is an almost direct product with

(a) $e_{\alpha} \in \operatorname{Lie}(S)$, and

(b) $S$ is almost simple of type $A_{1}$, and

(c) $\operatorname{Lie}(G)=\operatorname{Lie}(S) \oplus \operatorname{Lie}(H)$.

Our assumption on $p$ implies $(S \cap H)_{1} \subseteq \operatorname{Cent}(S)_{1}=e_{k}$, so that $(S \cap H)_{r}=e_{k}$. Thus, the canonical map $S \times H \longrightarrow G$ induces a closed embedding $S_{r} \times H_{r} \hookrightarrow G_{r}$. Applying [35, (I.9.6)] several times we obtain

$$
\operatorname{dim}_{k} k\left[G_{r}\right]=p^{r \operatorname{dim} G}=p^{r(\operatorname{dim} S+\operatorname{dim} H)}=\operatorname{dim}_{k} k\left[S_{r}\right] \operatorname{dim}_{k} k\left[H_{r}\right]=\operatorname{dim}_{k} k\left[S_{r} \times H_{r}\right],
$$

so that the above map is in fact an isomorphism.

In view of [35, (I.7.9(3))], the arguments of [22, (1.1)] show that the decomposition $G_{r}=S_{r} \times H_{r}$ induces an isomorphism

$$
Z_{r}(\lambda) \cong Z_{r}^{S}(\lambda) \otimes_{k} Z_{r}^{H}(\lambda)
$$

between $Z_{r}(\lambda)$ and the outer tensor product of the corresponding Verma modules of the factors. Thus, [51, (7.2)] implies

$$
\mathcal{V}_{G_{r}}\left(Z_{r}(\lambda)\right) \cong \mathcal{V}_{S_{r}}\left(Z_{r}^{S}(\lambda)\right) \times \mathcal{V}_{H_{r}}\left(Z_{r}^{H}(\lambda)\right)
$$

Since $e_{\alpha} \in \operatorname{Lie}(S)$, we see that $\alpha$ is a root of $S$. Consequently, $U_{\alpha} \subseteq S$, and another application of Corollary 5.2 implies that $\mathcal{V}_{\left(U_{\alpha}\right)_{r}}(k) \subseteq \mathcal{V}_{S_{r}}\left(Z_{r}^{S}(\lambda)\right)$. In particular, $2 \leq \operatorname{dim} \mathcal{V}_{S_{r}}\left(Z_{r}^{S}(\lambda)\right)$, so that $Z_{r}^{H}(\lambda)$ is projective. It thus follows from a consecutive application of [35, (II.11.8)] and [35, (II.9.6e)] that $Z_{r}^{H}(\lambda)$ is a simple projective $H_{r}$-module.

In view of [49, (7.2.4)], the group $S$ is isomorphic to $\mathrm{SL}(2)$ or $\operatorname{PSL}(2)$. Since $p \neq 2$, the center of $\mathrm{SL}(2)$ is reduced, so that the canonical map SL(2) $\longrightarrow \mathrm{PSL}(2)$ induces an isomorphism of the Frobenius kernels of these groups.

Let $L \subseteq G$ be a Levi subgroup of $G$ and $S \subseteq L$ be its semi-simple part. Given $\lambda \in X(T)$, we denote by $\Theta_{r}^{S} \overline{(\lambda)}$ the connected component of $\Gamma_{s}\left(S_{r}\right)$ containing the Verma module $Z_{r}^{S}\left(\left.\lambda\right|_{T \cap S}\right)$.

According to [35, (II.9.1(3), II.9.4, II.11.8)] a non-projective Verma module $Z_{r}(\lambda)$ is defined by a highest weight $\lambda$ of $\operatorname{depth} \operatorname{dp}(\lambda) \leq r$. The following theorem reduces us to the case $r \leq 2$. 
Theorem 7.4. Let $G$ be semi-simple and simply connected. Suppose that $p \geq 7$, and let $Z_{r}(\lambda)$ be a Verma module of complexity $\mathrm{cx}_{G_{r}}\left(Z_{r}(\lambda)\right)=2$. Then we have $r^{\prime}:=r-\operatorname{dp}(\lambda)+1 \leq 2$, and there exist a group $S \cong \mathrm{SL}(2), \mathrm{SL}(2) \times \mathrm{SL}(2), \mathrm{SL}(3)$, a weight $\gamma$ of $S$ of depth 1 , and an isomorphism $\Theta_{r}(\lambda) \cong \Theta_{r^{\prime}}(\gamma)$ sending $\left[Z_{r}(\lambda)\right]$ onto $\left[Z_{r^{\prime}}(\gamma)\right]$. Moreover, we have $S \cong \operatorname{SL}(2)$ for $r^{\prime}=2$.

Proof. We put $\operatorname{dp}(\lambda)=d+1$, with $d \geq 0$. Thanks to Theorem 6.6] and Corollary 6.7, there exists a weight $\mu$ of depth 1 and a quiver isomorphism $\Theta_{r}(\lambda) \cong \Theta_{r-d}(\mu)$, sending $\left[Z_{r}(\lambda)\right]$ onto $\left[Z_{r-d}(\mu)\right]$. By Theorem 6.6, we have

$$
r^{\prime}:=r-d \leq \operatorname{cx}_{G_{r-d}}\left(Z_{r-d}(\mu)\right)=\operatorname{cx}_{G_{r}}\left(Z_{r}(\lambda)\right)=2 .
$$

If $r^{\prime}=1$, then our result follows from [22, (3.5)]. Alternatively, Proposition 7.3 implies the existence of a decomposition $G_{r^{\prime}}=S_{r^{\prime}} \times H_{r^{\prime}}$ with $S \cong \mathrm{SL}(2)$. By the same token, there is an isomorphism

$$
Z_{r^{\prime}}(\mu) \cong Z_{r^{\prime}}^{S}(\mu) \otimes_{k} Z_{r^{\prime}}^{H}(\mu)
$$

with $Z_{r^{\prime}}^{H}(\mu)$ being a simple projective $H_{r^{\prime}}$-module. By the arguments of [22, (3.5)], the functor

$$
S_{r^{\prime}} \bmod \longrightarrow G_{r^{\prime}}-\bmod \quad ; \quad M \mapsto M \otimes_{k} Z_{r^{\prime}}^{H}(\mu)
$$

induces a Morita equivalence between the blocks $\mathcal{B}_{r^{\prime}}\left(\left.\mu\right|_{T \cap S}\right)$ and $\mathcal{B}_{r^{\prime}}(\mu)$ sending $Z_{r^{\prime}}^{S}(\mu)$ onto $Z_{r^{\prime}}(\mu)$. As a result, we obtain a quiver isomorphism $\Theta_{r^{\prime}}^{S}(\mu) \cong \Theta_{r^{\prime}}(\mu)$, which maps $\left[Z_{r^{\prime}}^{S}(\mu)\right]$ onto $\left[Z_{r^{\prime}}(\mu)\right]$. Our assertion thus follows by composing the above isomorphisms of stable translation quivers.

Remark. By the same line of arguments, a semi-simple, simply connected group $G$ affords Verma modules of complexity 2 only if it possesses almost simple factors of types $A_{1}$ or $A_{2}$.

Acknowledgements: This research was funded in part by EPSRC grant GR/R86546/01. Part of the research for this paper was carried out during a visit by the first author to the University of Southampton: he is grateful to the members of the School of Mathematics for their hospitality.

The authors would like to thank the referee for carefully reading the manuscript along with providing helpful suggestions.

\section{REFERENCES}

[1] J. Alperin and L. Evens, Representations, resolutions and Quillen's dimension theorem. J. Pure Appl. Algebra 22 (1981), 1-9.

[2] M. Auslander, I. Reiten, and S. Smalø, Representation Theory of Artin Algebras. Cambridge Studies in Advanced Mathematics 36. Cambridge University Press (1995).

[3] D. Benson, Representations and Cohomology, I. Cambridge Studies in Advanced Mathematics 30. Cambridge University Press, 1991.

[4] bysame, Representations and Cohomology, II. Cambridge Studies in Advanced Mathematics 31. Cambridge University Press, 1991.

[5] C. Bessenrodt, Modular representation theory for blocks with cyclic defect groups via the Auslander-Reiten quiver. J. Algebra 140 (1991), 247-262.

[6] _ The Auslander-Reiten quiver of a modular group algebra revisited. Math. Z. 206 (1991), 25-34.

[7] W. Bruns and J. Herzog, Cohen-Macaulay Rings. Cambridge Studies in Advanced Mathematics 39. Cambridge University Press, 1998.

[8] J. Carlson, Complexity and Krull dimension. Springer Lecture Notes in Mathematics 903 (1981), 62-67.

[9] _ The variety of an indecomposable module is connected. Invent. math. 77 (1984), 291-299.

[10] E. Cline, B. Parshall, and L. Scott, Finite dimensional algebras and highest weight categories. J. reine angew. Math. 391 (1988), 85-99.

[11] E. Cline, B. Parshall, L. Scott, and W. van der Kallen. Rational and generic cohomology. Invent. math. 39 (1977), 143-163. 
[12] M. Demazure and P. Gabriel, Groupes Algébriques. Masson, Paris/North Holland, Amsterdam 1970.

[13] K. Erdmann, Blocks of Tame Representation Type and Related Classes of Algebras. Lecture Notes in Mathematics 1428. Springer-Verlag, 1990.

[14] K. Erdmann and A. Skowroński, On Auslander-Reiten components of blocks and self-injective special biserial algebras. Trans. Amer. Math. Soc. 330 (1992), 165-189.

[15] R. Farnsteiner, Periodicity and representation type of modular Lie algebras. J. reine angew. Math. 464 (1995), 47-65.

[16] _ On support varieties of Auslander-Reiten components. Indag. Math. 10 (1999), 221-234.

[17] _ Auslander-Reiten components for Lie algebras of reductive groups. Adv. in Math. 155 (2000), $49-83$.

[18] _ On the Auslander-Reiten quiver of an infinitesimal group. Nagoya Math. J. 160 (2000), $103-121$.

[19] _ Auslander-Reiten components for $G_{1} T$-modules. J. Algebra and Appl. 4 (2005), 739-759.

$[20]$ - Group-graded algebras, extensions of infinitesimal groups, and applications. Transform. Groups 14 (2009), 127-162.

[21] Complexity, periodicity and one-parameter subgroups. Manuscript in preparation.

[22] R. Farnsteiner and G. Röhrle, Almost split sequences of Verma modules. Math. Ann. 322 (2002), 701-743.

[23] D. Fischman, S. Montgomery, and H. Schneider, Frobenius extensions of subalgebras of Hopf algebras. Trans. Amer. Math. Soc. 349 (1997), 4857-4895.

[24] E. Friedlander and B. Parshall, Support varieties for restricted Lie algebras. Invent. math. 86 (1986), 553-586.

[25] E. Friedlander and A. Suslin, Cohomology of finite group schemes over a field. Invent. math. 127 (1997), $209-270$.

[26] R. Gordon and E. Green, Graded Artin algebras. J. Algebra 76 (1982), 111-137.

[27] Representation theory of graded Artin algebras. J. Algebra 76 (1982), 138-152.

[28] D. Happel, Triangulated Categories in the Representation Theory of Finite Dimensional Algebras. LMS Lecture Note Series 119. Cambridge University Press, 1988.

[29] A. Heller, Indecomposable representations and the loop space operation. Proc. Amer. Math. Soc. 12 (1961), 640-643.

[30] J. Humphreys, Symmetry for finite dimensional Hopf algebras. Proc. Amer. Math. Soc. 68 (1978), $143-146$.

[31] _ Linear Algebraic Groups. Graduate Texts in Mathematics 21. Springer Verlag, 1981.

[32] J. Jantzen, Über Darstellungen höherer Frobenius-Kerne halbeinfacher algebraischer Gruppen. Math. Z. 164 (1979), 271-292.

[33] Support varieties of Weyl modules. Bull. London Math. Soc. 19 (1987), 238-244.

[34] _ Modular representations of reductive groups. J. Pure Appl. Algebra 152 (2000), $133-185$.

[35] — Representations of Algebraic Groups. Second edition. Mathematical Surveys and Monographs 107. American Mathematical Society, Providence, RI, 2003.

[36] T. Jost, On Specht modules in the Auslander-Reiten quiver. J. Algebra 173 (1995), 281-301.

[37] S. Kawata, On Auslander-Reiten components and simple modules for finite group algebras. Osaka J. Math. 34 (1997), 681-688.

[38] S. Kawata, G. Michler, and K. Uno, On simple modules in the Auslander-Reiten components of finite groups. Math. Z. 234 (2000), 375-398.

[39] _ On Auslander-Reiten components and simple modules for finite groups of Lie type. Osaka J. Math. 38 (2001), 21-26.

[40] R. Larson and M. Sweedler, An associative orthogonal form for Hopf algebras. Amer. J. Math. 91 (1969), 75-94.

[41] D. Nakano, B. Parshall, and D. Vella, Support varieties for algebraic groups. J. reine angew. Math. 547 (2002), 15-49.

[42] T. Okuyama, On the Auslander-Reiten quiver of a finite group. J. Algebra 110 (1987), 425-430.

[43] W. Pfautsch, Ext ${ }^{1}$ for the Frobenius kernels of $\mathrm{SL}_{2}$. Comm. Algebra 13 (1985), 169-179.

[44] C. Riedtmann, Algebren, Darstellungsköcher, Überlagerungen und zurück. Comment. Math. Helv. 55 (1980), 199-224.

[45] C. Ringel, Finite-dimensional hereditary algebras of wild representation type. Math. Z. 161 (1978), $235-255$.

[46] _ The category of modules with good filtrations over a quasi-hereditary algebra has almost split sequences. Math. Z. 208 (1991), 209-223.

[47] I. Reiten, Almost split sequences for group algebras of finite representation type. Trans. Amer. Math. Soc. 233 (1977), 125-136.

[48] J. Schue, Symmetry for the enveloping algebra of a restricted Lie algebra. Proc. Amer. Math. Soc. 16 (1965), $1123-124$.

[49] T. Springer, Linear Algebraic Groups. Progress in Mathematics 9. Birkhäuser-Verlag, Boston 1998.

[50] A. Suslin, E. Friedlander, and C. Bendel, Infinitesimal 1-parameter subgroups and cohomology. J. Amer. Math. Soc. 10 (1997), 693-728. 
[51] _ Support varieties for infinitesimal group schemes. J. Amer. Math. Soc. 10 (1997), 729-759.

[52] K. Uno, On the vertices of modules in the Auslander-Reiten quiver. Math. Z. 208 (1991), 411-436.

[53] W. Waterhouse, Introduction to Affine Group Schemes. Graduate Texts in Mathematics 66. Springer Verlag, 1979.

[54] C. Weibel, An Introduction to Homological Algebra. Cambridge Studies in Advanced Mathematics 38. Cambridge University Press, 1994.

[55] P. Webb, The Auslander-Reiten quiver of a finite group. Math. Z. 179 (1982), 97-121.

(R. Farnsteiner) Department of Mathematics, University of Kiel, Ludewig-Meyn-Str. 4, 24098 Kiel, GERMANY

E-mail address: rolf@math.uni-kiel.de

(G. Röhrle) Department of Mathematics, University of Bochum, 44780 Bochum, Germany

E-mail address: gerhard.roehrle@ruhr-uni-bochum.de 